\title{
THE DYNAMICS OF EQUATORIAL LONG WAVES: A SINGULAR LIMIT WITH FAST VARIABLE COEFFICIENTS*
}

\author{
ALEXANDRE DUTRIFOY ${ }^{\dagger}$ AND ANDREW MAJDA ${ }^{\ddagger}$
}

\begin{abstract}
At the equator, the Coriolis force from rotation vanishes identically so that multiple time scale dynamics for the equatorial shallow water equation naturally leads to singular limits of symmetric hyperbolic systems with fast variable coefficients. The classical strategy of using energy estimates for higher spatial derivatives has a fundamental difficulty since formally the commutator terms explode in the limit. Here this fundamental difficulty is circumvented by exploiting the special structure of the equatorial shallow water equations in suitable new variables involving the raising and lowering operators for the quantum harmonic oscillator, and obtaining uniform higher derivative estimates in a new function space based on the Hermite operator. The result is a completely new theorem characterizing the singular limit of the equatorial shallow water equations in the long wave regime, even with general unbalanced initial data, as a solution of the equatorial long wave equation. The results presented below point the way for rigorous PDE analysis of both the equatorial shallow water equations and the equatorial primitive equations in other physically relevant singular limit regimes.
\end{abstract}

Key words. Equatorial long wave equation, shallow water equations, singular limit, fast averaging.

AMS subject classifications. 35Q35, 76N10, 76U05, 76M45.

\section{Introduction}

Geophysical flows are a rich source of novel problems for applied mathematics and the contemporary theory of partial differential equations (PDE) ([16] and references therein). The reason for this is that many physically important geophysical flows involve complex nonlinear interaction over multi-scales in both time and space so developing simplified reduced models which are simpler yet capture key physical phenomena is of central importance $[8,23,24,16,18]$. In mid-latitudes, the fact that the rotational Coriolis terms are bounded away from zero leads to a strict temporal frequency scale separation between slow potential vorticity dynamics and fast gravity waves; this physical fact leads to new theorems justifying the quasi-geostrophic midlatitude dynamics even with general unbalanced initial data for both rapidly rotating shallow water equations and completely stratified flows $[3,5,6,17,16]$. The strategy in the above proofs is to adapt the classical framework of Klainerman and Majda for singular limits $[12,15,16]$ together with the important generalizations by Schochet $[25,26]$, which allow for fast wave averaging, to the dispersive systems of geophysical flows; it is well known that these theories require constant symmetric hyperbolic coefficients for the fast wave dynamics in order to obtain higher derivative estimates on the solution.

At the equator, the tangential projection of the Coriolis force from rotation vanishes identically so that there is no longer a time scale separation between potential vortical flows and gravity waves. This has profound consequences physically that allow the tropics to behave as a waveguide with extremely warm surface temperatures. The

\footnotetext{
*Received: Februay 9, 2006; accepted: March 30, 2006. Communicated by Shi Jin.

†Chargé de recherches du F. N. R. S. à l'Université Libre de Bruxelles, Campus de la Plaine, CP 214, Boulevard du Triomphe, 1050 Brussels, Belgium (adutrif@ulb.ac.be, http://homepages. vub.ac.be/ adutrif).

${ }^{\ddagger}$ Mathematics Department and Center for Atmosphere and Ocean Science, Courant Institute of Mathematical Sciences, New York University, New York, NY 10012, USA (jonjon@cims.nyu.edu, http://www.math.nyu.edu/faculty/majda/index.html).
} 
resulting behavior profoundly influences longer term mid-latitude weather prediction and climate change through hurricanes, monsoons, El Niño, and global teleconnections with the mid-latitude atmosphere. How this happens through detailed physical mechanisms is one of the most important contemporary problems in the atmosphere-ocean science community with a central role played by nonlinear interactive heating involving the interaction of clouds, moisture, and convection [24, 28, 20, 2, 19, 1, 7, 21]. The variable coefficient degeneracy of the Coriolis term at the equator alluded to earlier leads to both important new physical effects as well as fascinating new mathematical phenomena and PDE's [18, 20, 2, 19, 1, 7, 21]. Chapter 9 of ref. [16] provides an introduction to these topics for mathematicians while ref. [7] introduces and studies the simplest physical equatorial models with moisture. In this equatorial context, the new multi-scale reduced dynamical PDE models are even relatively recent in origin [18]. Thus, the need for additional PDE theory is very important for these disciplinary problems and this is the main topic of the present paper.

Formulation, theorems and strategy of proof

The simplest model for tropical dynamics is the shallow water equations for the equatorial region,

$$
\begin{aligned}
& \partial_{t} \vec{v}+\vec{v} \cdot \nabla \vec{v}+\epsilon^{-1}\left(y \vec{v}^{\perp}+\nabla h\right)=\left(S_{\epsilon}^{u}, S_{\epsilon}^{v}\right) \\
& \partial_{t} h+\vec{v} \cdot \nabla h+h \operatorname{div} \vec{v}+\epsilon^{-1} \operatorname{div} \vec{v}=S_{\epsilon}^{h}
\end{aligned}
$$

in which $\vec{v}=(u, v)(t, x, y)$ is the horizontal velocity, $h=h(t, x, y)$ the height, $x$ the longitude, $y$ the distance to the equator, $\vec{v}^{\perp}=(-v, u)$, and $S_{\epsilon}^{u}, S_{\epsilon}^{v}$, $S_{\epsilon}^{h}$ forcing terms which we regard as known in the present paper. In (1.1), the equatorial shallow water equations have been written in natural equatorial units of space and time under the non-dimensional assumptions that the typical fluid velocity magnitude ratio to the gravity wave speed, the Froude number, is of order $\epsilon$ and also the height fluctuations are order $\epsilon$ (see[18], pp. 394-395). As discussed in [18] and [16] (see pp. 220-221), it is also interesting to study long wave solutions of (1.1) in the $x$-direction, i.e. solutions $(\vec{v}, h)(\epsilon x, y, t)$. Rescaling (1.1) in the horizontal variable $\left(x^{\prime}=\epsilon x\right)$ and dropping the primes gives the scaled equatorial shallow water equations

$$
\begin{aligned}
& \partial_{t} u+\epsilon u \partial_{x} u+v \partial_{y} u+\partial_{x} h-\epsilon^{-1} y v=S_{\epsilon}^{u} \\
& \partial_{t} v+\epsilon u \partial_{x} v+v \partial_{y} v+\epsilon^{-1}\left(y u+\partial_{y} h\right)=S_{\epsilon}^{v} \\
& \partial_{t} h+\epsilon u \partial_{x} h+v \partial_{y} h+(1+\epsilon h) \partial_{x} u+h \partial_{y} v+\epsilon^{-1} \partial_{y} v=S_{\epsilon}^{h} .
\end{aligned}
$$

We study these equations for $(x, y) \in \mathbb{T} \times \mathbb{R}$ and $\epsilon>0$ tending to 0 , supplementing them with initial conditions

$$
\left.u\right|_{t=0}=u_{0 \epsilon},\left.\quad v\right|_{t=0}=v_{0 \epsilon},\left.\quad h\right|_{t=0}=h_{0 \epsilon} .
$$

This is a singular limit of a symmetric hyperbolic system with a fast variable coefficient created by the degenerate Coriolis terms involving $y$ in $\left(\mathrm{SW}_{\epsilon}\right)$. Few other systems with fast variable coefficients have been treated previously (one example is Section 4 in [10]). In rough outline, the proof below follows the classical strategy for singular limits $[11,12,15,25,26]$ which immediately presents a fundamental difficulty; straightforwardly differentiating $\left(\mathrm{SW}_{\epsilon}\right)$ with respect to $y$ and doing energy estimates leads to terms with magnitude $O\left(\epsilon^{-1}\right)$ from the commutators which formally can blow up as $\epsilon \downarrow 0$. The key new ideas in the paper involve how to circumvent this difficulty through a different route using the physical structure of $\left(\mathrm{SW}_{\epsilon}\right)$. Next, the main results and the strategy of the proof are summarized briefly. 
We first show that the family of initial value problems $\left(\mathrm{SW}_{\epsilon}, \mathrm{IC}_{\epsilon}\right)$ has strong solutions defined on a time-interval independent of $\epsilon$ if the data are bounded in a suitably modified Sobolev space.

Definition 1.1. For $s \in \mathbb{N}$, we denote by $\tilde{W}^{s}$ the space of functions $f \in L^{2}(\mathbb{T} \times \mathbb{R})$ such that

$$
\sum_{\alpha+\beta+\gamma \leq s}\left\|\partial_{x}^{\alpha} y^{\beta} \partial_{y}^{\gamma} f\right\|_{L^{2}}<\infty
$$

THEOREM 1.2. If $\left\|\left(u_{0 \epsilon}, v_{0 \epsilon}, h_{0 \epsilon}\right)\right\|_{\tilde{W}^{4}} \leq C$ and $\left\|\vec{S}_{\epsilon}\right\|_{C\left(\mathbb{R} ; \tilde{W}^{4}\right)} \leq C$ for some constant $C$ independent of $\epsilon$, there is a time $T>0$ such that solutions $\left(u_{\epsilon}, v_{\epsilon}, h_{\epsilon}\right)$ of $\left(S W_{\epsilon}, I C_{\epsilon}\right)$ exist (and are unique) in $C\left([0, T] ; \tilde{W}^{4}\right) \cap C^{1}\left([0, T] ; \tilde{W}^{3}\right)$ for all $\epsilon$.

Next, using Schochet's method of fast averaging [25], we will decompose these solutions into two parts: a fast oscillating part tending weakly to zero, and a slow part, involving only $u_{\epsilon}$ and $h_{\epsilon}$, converging strongly to the solution $(u, h)$ of the Linear Equatorial Long-Wave System:

$$
\left\{\begin{array}{l}
\partial_{t} u+\partial_{x} h-y v=\tilde{S}^{u} \\
\partial_{t} h+\partial_{x} u+\partial_{y} v=\tilde{S}^{h} \\
y u+\partial_{y} h=0 \\
\left.u\right|_{t=0}=u_{0},\left.\quad h\right|_{t=0}=h_{0},
\end{array}\right.
$$

provided that the initial data and forcing terms converge accordingly. In (LW), the variable $v$ plays the role of a Lagrange multiplier which can be eliminated from the equations using the condition $y u+\partial_{y} h=0$, in the same way as the pressure can be eliminated from the incompressible Euler equations using the condition of free divergence [16]. The linear equatorial long wave equation (LW) has central importance both for theories and prediction of El Niño in the equatorial ocean [24] and for atmospheric wave dynamics in the tropics $[18,20,2,19]$.

Let $\tilde{P}_{0}$ denote the orthogonal projector, in $\left(L^{2}(\mathbb{T} \times \mathbb{R})\right)^{3}$, onto vector fields $(u, v, h)$ such that $y u+\partial_{y} h=0$ and $v=0$. Here is the main theorem.

THEOREM 1.3. Assume that the hypotheses of Theorem 1.2 are satisfied and let $\left(u_{\epsilon}, v_{\epsilon}, h_{\epsilon}\right)$ be the solutions of $\left(S W_{\epsilon}, I C_{\epsilon}\right)$ this theorem provides on $[0, T]$.

Assume, in addition, that $\left(u_{0 \epsilon}, v_{0 \epsilon}, h_{0 \epsilon}\right)$ and the forcing terms $\vec{S}_{\epsilon}(t)$ converge respectively to $\left(u_{0}, v_{0}, h_{0}\right)$ and $\vec{S}(t)$ in $L^{2}$, uniformly in $t$, and that $\left\|\vec{S}_{\epsilon}\right\|_{\operatorname{Lip}\left([0, T] ; L^{2}\right)} \leq C$.

Then there is weak convergence $\left(u_{\epsilon}, v_{\epsilon}, h_{\epsilon}\right) \rightarrow(u, 0, h)$, and even strong convergence $\tilde{P}_{0}\left(u_{\epsilon}, v_{\epsilon}, h_{\epsilon}\right) \rightarrow(u, 0, h)$ in $C\left([0, T] ; \tilde{W}^{s}\right)$ for all $s<4$, where $(u, h)$ is the solution of $(L W)$ with $\tilde{S}^{u}=\left(\tilde{P}_{0} \vec{S}\right)^{u}$ and $\tilde{S}^{h}=\left(\tilde{P}_{0} \vec{S}\right)^{h}$.

These results rely mainly on a key a priori estimate in $\tilde{W}^{4}$, presented in Section 3, uniform with respect to $\epsilon$, for the solutions of $\left(\mathrm{SW}_{\epsilon}, \mathrm{IC}_{\epsilon}\right)$. To get this estimate and also to be able to do the averaging, we rewrite the equations (see next section) as

$$
\left\{\begin{array}{l}
\partial_{t} \vec{U}+S_{1} \partial_{x} \vec{U}+S_{2} \partial_{y} \vec{U}+\frac{1}{\epsilon} L \vec{U}=\vec{F}_{\epsilon}, \\
\left.\vec{U}\right|_{t=0}=\vec{U}_{0, \epsilon}
\end{array}\right.
$$

where $S_{1}, S_{2}$ are symmetric matrices depending linearly on $\vec{U}$ and

$$
L=\frac{1}{\sqrt{2}}\left(\begin{array}{ccc}
0 & 0 & L_{-} \\
0 & 0 & L_{+} \\
L_{+} & L_{-} & 0
\end{array}\right)
$$


in which $L_{+}$and $L_{-}$denote the classical lowering and raising operators of mathematical physics:

$$
L_{ \pm}=\partial_{y} \pm y \text {. }
$$

It is then natural to decompose the system along parabolic cylinder functions (Section 2.2). We already knew that (LW) could be completely solved by this method [16]. In the present context, the decomposition will motivate our choice of functional space (Section 2.3) and make it easy to build a basis of $\left(L^{2}\right)^{3}$ with eigenvectors of the fast-wave operator $L$ (Section 2.4), allowing us to define the fast-wave and slow-wave projectors (Section 2.5). Theorems 1.2 and 1.3 are finally restated as Theorems 2.5 and 2.6 in the new notation (Section 2.6). The detailed proofs are given in Section 3, which begins with the key a priori estimate.

Finally, we emphasize here that one surprising consequence of the analysis below is the strong convergence of $\tilde{P}_{0}\left(u_{\epsilon}, v_{\epsilon}, h_{\epsilon}\right)$ to $(u, 0, h)$ with general unbalanced initial data; this property is not true for many other singular limits.

\section{Reformulation of the equations}

2.1. Changes of variables. The first step is to make the system symmetric by setting

$$
\tilde{h}=\frac{2 h}{1+\sqrt{1+\epsilon h}},
$$

which is equivalent to

$$
\frac{\tilde{h}}{2}=\frac{\sqrt{1+\epsilon h}-1}{\epsilon}
$$

or

$$
1+\epsilon h=\left(1+\frac{1}{2} \epsilon \tilde{h}\right)^{2}
$$

so that

$$
\left(\partial_{t}, \partial_{x}, \partial_{y}\right) h=\left(1+\frac{1}{2} \epsilon \tilde{h}\right)\left(\partial_{t}, \partial_{x}, \partial_{y}\right) \tilde{h}
$$

Thus the equations $\left(\mathrm{SW}_{\epsilon}\right)$ becomes

$$
\begin{aligned}
& \partial_{t} u+\epsilon u \partial_{x} u+v \partial_{y} u+\left(1+\frac{1}{2} \epsilon \tilde{h}\right) \partial_{x} \tilde{h}-\epsilon^{-1} y v=S_{\epsilon}^{u} \\
& \partial_{t} v+\epsilon u \partial_{x} v+v \partial_{y} v+\frac{1}{2} \tilde{h} \partial_{y} \tilde{h}+\epsilon^{-1}\left(y u+\partial_{y} \tilde{h}\right)=S_{\epsilon}^{v} \\
& \partial_{t} \tilde{h}+\epsilon u \partial_{x} \tilde{h}+v \partial_{y} \tilde{h}+\left(1+\frac{1}{2} \epsilon \tilde{h}\right) \partial_{x} u+\frac{1}{2} \tilde{h} \partial_{y} v+\epsilon^{-1} \partial_{y} v=S_{\epsilon}^{\tilde{h}}
\end{aligned}
$$

with

$$
S_{\epsilon}^{\tilde{h}}=\frac{S_{\epsilon}^{h}}{1+\frac{1}{2} \epsilon \tilde{h}} .
$$

This trick is exactly the same as the one which enables us to write the compressible Euler equations in a symmetric form [11, 12, 27]. 
Now, if we drop the nonlinear terms in (2.2a) and (2.2c), we are left with $\partial_{t} u+\partial_{x} \tilde{h}$ in the first equation and $\partial_{t} \tilde{h}+\partial_{x} u$ in the second. This suggests the orthogonal change of variables

$$
r=\frac{1}{\sqrt{2}}(u+\tilde{h}), \quad l=\frac{1}{\sqrt{2}}(-u+\tilde{h}),
$$

which gives

$$
\begin{aligned}
& \partial_{t} r+\left(1+\frac{\epsilon}{2 \sqrt{2}}(3 r-l)\right) \partial_{x} r+ v \partial_{y} r+\frac{1}{4}(r+l) \partial_{y} v \\
&+\epsilon^{-1} \frac{1}{\sqrt{2}}\left(\partial_{y} v-y v\right)=F_{\epsilon}^{1} \\
& \partial_{t} l+\left(-1+\frac{\epsilon}{2 \sqrt{2}}(r-3 l)\right) \partial_{x} l+ v \partial_{y} l+\frac{1}{4}(r+l) \partial_{y} v \\
&+\epsilon^{-1} \frac{1}{\sqrt{2}}\left(\partial_{y} v+y v\right)=F_{\epsilon}^{2} \\
& \partial_{t} v+\frac{\epsilon}{\sqrt{2}}(r-l) \partial_{x} v+v \partial_{y} v+\frac{1}{4}(r+l) \partial_{y} r+\frac{1}{4}(r+l) \partial_{y} l \\
&+\epsilon^{-1} \frac{1}{\sqrt{2}}\left(\partial_{y} r+y r+\partial_{y} l-y l\right)=F_{\epsilon}^{3},
\end{aligned}
$$

with

$$
F_{\epsilon}^{1}=\frac{1}{\sqrt{2}}\left(S_{\epsilon}^{u}+S_{\epsilon}^{\tilde{h}}\right), \quad F_{\epsilon}^{2}=\frac{1}{\sqrt{2}}\left(-S_{\epsilon}^{u}+S_{\epsilon}^{\tilde{h}}\right), \quad F_{\epsilon}^{3}=S_{\epsilon}^{v}
$$

So, introducing the vector

$$
\vec{U}=\left(\begin{array}{c}
r \\
l \\
v
\end{array}\right)
$$

we get (1.2) with

$$
\begin{aligned}
& S_{1}=S_{1}^{0}+\epsilon S_{1}^{1}, \\
& S_{1}^{0}=\left(\begin{array}{ccc}
1 & 0 & 0 \\
0 & -1 & 0 \\
0 & 0 & 0
\end{array}\right), \\
& S_{1}^{1}=S_{1}^{1}(\vec{U})=\frac{1}{2 \sqrt{2}}\left(\begin{array}{ccc}
3 r-l & 0 & 0 \\
0 & r-3 l & 0 \\
0 & 0 & 2 r-2 l
\end{array}\right)
\end{aligned}
$$

and

$$
S_{2}=S_{2}(\vec{U})=\frac{1}{4}\left(\begin{array}{ccc}
4 v & 0 & r+l \\
0 & 4 v & r+l \\
r+l & r+l & 4 v
\end{array}\right)
$$


2.2. Decomposition of the system using parabolic cylinder functions.

The operators $L_{ \pm}$are called lowering and raising because of their action on the parabolic cylinder functions:

$$
\begin{aligned}
\frac{1}{\sqrt{2}} L_{-} \phi_{n} & =-\sqrt{n+1} \phi_{n+1} \quad \text { for } n \geq 0, \\
\frac{1}{\sqrt{2}} L_{+} \phi_{n} & =\sqrt{n} \phi_{n-1} \quad \text { for } n \geq 1, \\
L_{+} \phi_{0} & =0,
\end{aligned}
$$

where

$$
\phi_{n}(y)=\left(2^{n} n ! \sqrt{\pi}\right)^{-1 / 2} H_{n}(y) e^{-\frac{y^{2}}{2}}, \quad n=0,1, \ldots
$$

in which

$$
H_{n}(y)=(-1)^{n} e^{y^{2}} \frac{d^{n}}{d y^{n}} e^{-y^{2}}
$$

denotes the Hermite polynomial of degree $n$.

Since the $\phi_{n}$ form an orthonormal basis of $L^{2}(\mathbb{R})$, we may decompose the components of $\vec{U}$ as follows:

$$
\begin{aligned}
& r(t, x, y)=\sum_{n=0}^{\infty} r_{n}(t, x) \phi_{n}(y), \\
& l(t, x, y)=\sum_{n=0}^{\infty} l_{n}(t, x) \phi_{n}(y), \\
& v(t, x, y)=\sum_{n=0}^{\infty} v_{n}(t, x) \phi_{n}(y) .
\end{aligned}
$$

From (1.3) and (2.8), it is readily seen that $L$ acts for each $t$ and $x$ inside finitedimensional subspaces of $\left(L^{2}(\mathbb{R})\right)^{3}$ : first,

$$
L\left(\begin{array}{c}
\phi_{0} \\
0 \\
0
\end{array}\right)=0
$$

second,

$$
L\left(\begin{array}{c}
\phi_{1} \\
0 \\
0
\end{array}\right)=\left(\begin{array}{c}
0 \\
0 \\
\phi_{0}
\end{array}\right) \quad \text { and } \quad L\left(\begin{array}{c}
0 \\
0 \\
\phi_{0}
\end{array}\right)=-\left(\begin{array}{c}
\phi_{1} \\
0 \\
0
\end{array}\right)
$$

third, for all $n \geq 0$,

$$
\begin{aligned}
L\left(\begin{array}{c}
\phi_{n+2} \\
0 \\
0
\end{array}\right) & =\sqrt{n+2}\left(\begin{array}{c}
0 \\
0 \\
\phi_{n+1}
\end{array}\right), \\
L\left(\begin{array}{c}
0 \\
\phi_{n} \\
0
\end{array}\right) & =-\sqrt{n+1}\left(\begin{array}{c}
0 \\
0 \\
\phi_{n+1}
\end{array}\right),
\end{aligned}
$$


and

$$
L\left(\begin{array}{c}
0 \\
0 \\
\phi_{n+1}
\end{array}\right)=-\sqrt{n+2}\left(\begin{array}{c}
\phi_{n+2} \\
0 \\
0
\end{array}\right)+\sqrt{n+1}\left(\begin{array}{c}
0 \\
\phi_{n} \\
0
\end{array}\right) .
$$

Now consider $\left(L^{2}(\mathbb{R} \times \mathbb{T})\right)^{3}$ equipped with the usual scalar (hermitian) product

$$
\langle\vec{V}, \vec{W}\rangle=\int_{\mathbb{T}} \int_{\mathbb{R}} \vec{V}(x, y) \cdot \overline{\vec{W}(x, y)} d x d y .
$$

Let $P^{(-2)}, P^{(-1)}$, and $P^{(n)}$ for $n \geq 0$, be the orthogonal projections onto its subspaces generated by ${ }^{t}\left(\phi_{0}, 0,0\right)$, by ${ }^{t}\left(\phi_{1}, 0,0\right)$ and ${ }^{t}\left(0,0, \phi_{0}\right)$, and by ${ }^{t}\left(\phi_{n+2}, 0,0\right),{ }^{t}\left(0, \phi_{n}, 0\right)$ and ${ }^{t}\left(0,0, \phi_{n+1}\right)$.

Equations (2.9) to (2.13) imply that $L$ and $P^{(n)}$ commute for all $n$; therefore, (1.2) is equivalent to the family of systems

$$
\left\{\begin{array}{l}
\partial_{t} P^{(n)} \vec{U}+P^{(n)}\left(S_{1} \partial_{x} \vec{U}+S_{2} \partial_{y} \vec{U}\right)+\frac{1}{\epsilon} L P^{(n)} \vec{U}=P^{(n)} \vec{F}_{\epsilon} \\
\left.P^{(n)} \vec{U}\right|_{t=0}=P^{(n)} \vec{U}_{0, \epsilon}
\end{array}\right.
$$

for $n \geq-2$.

2.3. The functional spaces $\tilde{W}^{s}$. When we take the scalar product of $(2.14)$ with $P^{(n)} \vec{U}$, the term in $\epsilon^{-1}$ disappears. Indeed, integration by parts shows that $L$ is skew-symmetric in $\left(L^{2}\right)^{3}$, so $\left\langle L P^{(n)} \vec{U}, P^{(n)} \vec{U}\right\rangle=0$. We can also check it explicitly:

$$
\begin{aligned}
\left\langle P^{(n)} \vec{U}, L P^{(n)} \vec{U}\right\rangle & \\
= & \left\langle\left(\begin{array}{c}
r_{n+2} \phi_{n+2} \\
l_{n} \phi_{n} \\
v_{n+1} \phi_{n+1}
\end{array}\right),\left(\begin{array}{c}
-v_{n+1} \sqrt{n+2} \phi_{n+2} \\
v_{n+1} \sqrt{n+1} \phi_{n} \\
r_{n+2} \sqrt{n+2} \phi_{n+1}-l_{n} \sqrt{n+1} \phi_{n+1}
\end{array}\right)\right\rangle \\
= & -\int_{\mathbb{T}} r_{n+2}(t, x) v_{n+1}(t, x) \sqrt{n+2} d x \int_{\mathbb{R}} \phi_{n+2}^{2}(y) d y \\
& +\int_{\mathbb{T}} l_{n}(t, x) v_{n+1}(t, x) \sqrt{n+1} d x \int_{\mathbb{R}} \phi_{n}^{2}(y) d y \\
& +\int_{\mathbb{T}} v_{n+1}(t, x) r_{n+2}(t, x) \sqrt{n+2} d x \int_{\mathbb{R}} \phi_{n+1}^{2}(y) d y \\
& -\int_{\mathbb{T}} v_{n+1}(t, x) l_{n}(t, x) \sqrt{n+1} d x \int_{\mathbb{R}} \phi_{n+1}^{2}(y) d y \\
= & 0,
\end{aligned}
$$

because $\int_{\mathbb{R}} \phi_{n}^{2}(y) d y=1$ for all $n$, so that (2.17) cancels (2.15) and (2.18) cancels (2.16).

So, for any function $\varphi,(2.14)$ implies

$$
\begin{gathered}
\frac{1}{2} \partial_{t}\left(\left\|\varphi(n) P^{(n)} \vec{U}\right\|_{L^{2}}^{2}\right)+\varphi^{2}(n)\left\langle P^{(n)}\left(S_{1} \partial_{x} \vec{U}+S_{2} \partial_{y} \vec{U}\right), P^{(n)} \vec{U}\right\rangle \\
=\left\langle\varphi(n) P^{(n)} \vec{F}_{\epsilon}, \varphi(n) P^{(n)} \vec{U}\right\rangle
\end{gathered}
$$

for all $n \geq 2$. By summing over $n$, this gives a uniform estimate on

$$
\left(\sum_{n=-2}^{\infty}\left\|\varphi(n) P^{(n)} \vec{U}\right\|_{L^{2}}^{2}\right)^{1 / 2}
$$


if the term $\sum_{n=-2}^{\infty} \varphi^{2}(n)\left\langle P^{(n)}\left(S_{1} \partial_{x} \vec{U}+S_{2} \partial_{y} \vec{U}\right), P^{(n)} \vec{U}\right\rangle$ can be disposed of.

In view of (2.8), the derivation with respect to $y$ and the multiplication by $y$ both roughly amount to $\sum_{n=-2}^{+\infty} \sqrt{n} P^{(n)}$. This motivates our definition of modified Sobolev spaces $\tilde{W}^{s}$ below. As we explain in more detail in Section 3.1, the estimate in $\tilde{W}^{4}$ is obtained by choosing $\varphi(n) \sim n^{2}$.

Definition 2.1. We denote by $\tilde{W}^{s}$ the subspace of $L^{2}(\mathbb{T} \times \mathbb{R})$ consisting of functions

$$
f(x, y)=\sum_{k=-\infty}^{+\infty} \sum_{n=0}^{\infty} f_{k n} e^{2 \pi i k x} \phi_{n}(y)
$$

whose norm

$$
\|f\|_{\tilde{W}^{s}}=\left(\sum_{k=-\infty}^{+\infty} \sum_{n=0}^{\infty}(1+|k|+\sqrt{n})^{2 s}\left|f_{k n}\right|^{2}\right)^{1 / 2}
$$

is finite.

The next proposition implies in particular that Definition 2.1 agrees with Definition 1.1 for all values of $s$. It also shows that the role played by $\partial_{y}$ in a usual Sobolev space $W^{s}$ is played here by both $\partial_{y}$ and $y$, or $L_{-}$alone, or $L_{+}$alone.

Proposition 2.2. For $s \in \mathbb{N}$ and $f \in L^{2}(\mathbb{T} \times \mathbb{R})$, the norms

$$
\begin{aligned}
& \sum_{\alpha+\beta+\gamma \leq s}\left\|\partial_{x}^{\alpha} y^{\beta} \partial_{y}^{\gamma} f\right\|_{L^{2}}, \\
& \left\|\partial_{x}^{s} f\right\|_{L^{2}}+\left\|L_{-}^{s} f\right\|_{L^{2}} \\
& \left\|\partial_{x}^{s} f\right\|_{L^{2}}+\left\|L_{+}^{s} f\right\|_{L^{2}}+\|f\|_{L^{2}}
\end{aligned}
$$

are all equivalent to (NO).

Proof. • (N1) $\lesssim(\mathrm{N} 0)$. Indeed,

$$
\partial_{x}^{\alpha} y^{\beta} \partial_{y}^{\gamma} f=\frac{1}{4} \sum_{k=-\infty}^{+\infty} \sum_{n=0}^{+\infty} f_{k n}(2 \pi i k)^{\alpha} e^{2 \pi i k x}\left(L_{+}-L_{-}\right)^{\beta}\left(L_{+}+L_{-}\right)^{\gamma} \phi_{n} ;
$$

by $(2.8)$,

$$
\left(L_{+}-L_{-}\right)^{\beta}\left(L_{+}+L_{-}\right)^{\gamma} \phi_{n}=\sum_{l=-\beta-\gamma}^{\beta+\gamma} c_{n l} \phi_{n+l}
$$

for some coefficients $c_{n l} \leq C(1+\sqrt{n})^{\beta+\gamma}$; hence

$$
\begin{aligned}
\left\|\partial_{x}^{\alpha} y^{\beta} \partial_{y}^{\gamma} f\right\|_{L^{2}} & \leq \frac{C}{4}(2 \pi)^{\alpha} \sum_{l=-\beta-\gamma}^{\beta+\gamma}\left\|\sum_{k=-\infty}^{+\infty} \sum_{n=0}^{+\infty} f_{k n}|k|^{\alpha}(1+\sqrt{n})^{\beta+\gamma} \phi_{n+l}\right\|_{L^{2}} \\
& \leq \frac{C}{2}(2 \pi)^{\alpha}\left(\beta+\gamma+\frac{1}{2}\right)\|f\|_{\tilde{W}^{s}}
\end{aligned}
$$

if $\alpha+\beta+\gamma \leq s$, because then $|k|^{\alpha}(1+\sqrt{n})^{\beta+\gamma} \leq(1+|k|+\sqrt{n})^{s}$.

- $(\mathrm{N} 2) \lesssim(\mathrm{N} 1)$ and $(\mathrm{N} 3) \lesssim(\mathrm{N} 1)$ are trivial (just replace $L_{ \pm}$by $\left.\partial_{y} \pm y\right)$. 
- $(\mathrm{N} 0) \lesssim(\mathrm{N} 2)$ follows from

$$
\left\|\partial_{x}^{s} f\right\|_{L^{2}}=(2 \pi)^{s}\left(\sum_{k=-\infty}^{+\infty} \sum_{n=0}^{+\infty}|k|^{2 s}\left|f_{k n}\right|^{2}\right)^{1 / 2}
$$

and

$$
\begin{aligned}
\left\|L_{-}^{s} f\right\|_{L^{2}} & =\left\|\sum_{k=-\infty}^{+\infty} \sum_{n=0}^{+\infty} f_{k n} e^{2 \pi i k x}\left(\prod_{l=1}^{s}-\sqrt{2} \sqrt{n+l}\right) \phi_{n+s}\right\|_{L^{2}} \\
& \geq\left(\sum_{k=-\infty}^{+\infty} \sum_{n=0}^{+\infty}(1+\sqrt{n})^{2 s}\left|f_{k n}\right|^{2}\right)^{1 / 2}
\end{aligned}
$$

- $(\mathrm{N} 0) \lesssim(\mathrm{N} 3)$ follows from

$$
\begin{aligned}
\left\|L_{+}^{s} f\right\|_{L^{2}} & =\left\|\sum_{k=-\infty}^{+\infty} \sum_{n=s}^{+\infty} f_{k n} e^{2 \pi i k x}\left(\prod_{l=0}^{s-1} \sqrt{2} \sqrt{n-l}\right) \phi_{n-s}\right\|_{L^{2}} \\
& \geq C_{s}\left(\sum_{k=-\infty}^{+\infty} \sum_{n=s}^{+\infty}(1+\sqrt{n})^{2 s}\left|f_{k n}\right|^{2}\right)^{1 / 2}
\end{aligned}
$$

where $C_{s}$ is such that $\sqrt{n-s+1} \geq C_{s}(1+\sqrt{n})$ for all $n \geq s$, and

$$
\|f\|_{L^{2}} \geq \frac{1}{(1+\sqrt{s-1})^{s}}\left(\sum_{k=-\infty}^{+\infty} \sum_{n=0}^{s-1}(1+\sqrt{n})^{2 s}\left|f_{k n}\right|^{2}\right)^{1 / 2},
$$

and (2.20) again.

Corollary 2.3. For $s \in \mathbb{N}, \tilde{W}^{s}$ is embedded in the usual Sobolev space $W^{s}$. In particular, $\tilde{W}^{4} \subset C^{1}$.

Proof. The norm in $W^{s}$ is smaller than (N1).

Finally let $H$ be the Hermite operator, defined by

$$
H f=\frac{1}{2}\left(L_{+} L_{-}+L_{-} L_{+}\right) f=\frac{d^{2}}{d y^{2}} f-y^{2} f .
$$

Recall that

$$
H \phi_{n}=-(2 n+1) \phi_{n}
$$

for all $n \geq 0$.

Proposition 2.4. For s even, the norm in $\tilde{W}^{s}$ is equivalent to

$$
\left\|\partial_{x}^{s} f\right\|_{L^{2}}+\left\|H^{s / 2} f\right\|_{L^{2}} .
$$


Proof. Because of (2.21),

$$
\begin{aligned}
\left\|H^{s / 2} f\right\|_{L^{2}} & =\left\|\sum_{k=-\infty}^{+\infty} \sum_{n=0}^{+\infty} f_{k n} e^{2 \pi i k x}(2 n+1)^{s / 2} \phi_{n}\right\|_{L^{2}} \\
& \geq C\left(\sum_{k=-\infty}^{+\infty} \sum_{n=0}^{+\infty}(1+\sqrt{n})^{2 s}\left|f_{k n}\right|^{2}\right)^{1 / 2} .
\end{aligned}
$$

So $(\mathrm{N} 0) \lesssim(\mathrm{N} 4)$ by $(2.20)$ and $(2.22)$.

Again, (N4) $\lesssim$ (N1) is immediate when $H$ is replaced by its definition.

2.4. The eigenvalues and eigenvectors of $L$. Since $i L$ is self-adjoint on $\left(L^{2}\right)^{3}$, the eigenvectors of $L$ must be mutually orthogonal. We set

$$
\vec{e}_{0}^{-1}=\left(\begin{array}{c}
\phi_{0} \\
0 \\
0
\end{array}\right)
$$

by $(2.9), L \vec{e}_{0}^{-1}=0$. By $(2.10)$,

$$
\vec{e}_{ \pm 1}^{-1}=\frac{1}{\sqrt{2}}\left(\begin{array}{c} 
\pm i \phi_{1} \\
0 \\
\phi_{0}
\end{array}\right)
$$

are eigenvectors of $L$ corresponding to the eigenvalues $\pm i$, respectively. Finally, a short calculation on equations (2.11), (2.12), (2.13) yields the eigenvectors

$$
\vec{e}_{0}^{n}=\frac{1}{\sqrt{2 n+3}}\left(\sqrt{n+1}\left(\begin{array}{c}
\phi_{n+2} \\
0 \\
0
\end{array}\right)+\sqrt{n+2}\left(\begin{array}{c}
0 \\
\phi_{n} \\
0
\end{array}\right)\right),
$$

corresponding to the eigenvalue 0 , and

$$
\begin{gathered}
\vec{e}_{ \pm 1}^{n}=\frac{1}{\sqrt{2}} \frac{1}{\sqrt{2 n+3}}\left( \pm i \sqrt{n+2}\left(\begin{array}{c}
\phi_{n+2} \\
0 \\
0
\end{array}\right) \mp i \sqrt{n+1}\left(\begin{array}{c}
0 \\
\phi_{n} \\
0
\end{array}\right)\right. \\
\left.+\sqrt{2 n+3}\left(\begin{array}{c}
0 \\
0 \\
\phi_{n+1}
\end{array}\right)\right)
\end{gathered}
$$

corresponding to the eigenvalues $\pm i \sqrt{2 n+3}$.

The $\vec{e}_{\alpha}^{n}$ span the whole of $L^{2}(\mathbb{R})$ and we have

$$
L \vec{e}_{\alpha}^{n}=\alpha i \sqrt{2 n+3} \vec{e}_{\alpha}^{n}
$$

for all $n \geq-1$ and $\alpha=0, \pm 1$.

2.5. Projectors. We define $P_{0}, P_{1}, P_{-1}, P_{0}^{(n)}, P_{1}^{(n)}$ and $P_{-1}^{(n)}$ for $n \geq-1$ by

$$
\begin{array}{ll}
\left(P_{0}^{(n)} \vec{U}\right)(x, y)=\left(\int_{\mathbb{R}} \vec{U}(x, y) \cdot \vec{e}_{0}^{n}\left(y^{\prime}\right) d y^{\prime}\right) \vec{e}_{0}^{n}(y), & P_{0}=\sum_{n=0}^{\infty} P_{0}^{(n)}, \\
\left(P_{ \pm 1}^{(n)} \vec{U}\right)(x, y)=\left(\int_{\mathbb{R}} \vec{U}\left(x, y^{\prime}\right) \cdot \overline{\vec{e}_{ \pm 1}^{n}\left(y^{\prime}\right)} d y^{\prime}\right) \vec{e}_{ \pm 1}^{n}(y), & P_{ \pm 1}=\sum_{n=0}^{\infty} P_{ \pm 1}^{(n)} .
\end{array}
$$


We also write $P_{\text {osc }}=P_{1}+P_{-1}$. Remark that

$$
\begin{aligned}
& P^{(-2)}=P_{0}^{(-1)} \\
& P^{(-1)}=P_{1}^{(-1)}+P_{-1}^{(-1)}
\end{aligned}
$$

and

$$
P^{(n)}=P_{0}^{(n)}+P_{1}^{(n)}+P_{-1}^{(n)}
$$

for all $n \geq 0$.

2.6. Statements and comments. Our results can now be restated as follows.

Theorem 2.5 (existence). If $\left\|\vec{U}_{0, \epsilon}\right\|_{\tilde{W}^{4}} \leq C$ and $\left\|\vec{F}_{\epsilon}\right\|_{C\left(\mathbb{R} ; \tilde{W}^{4}\right)} \leq C$ for some constant $C$ independent of $\epsilon$, there is a time $T>0$ such that solutions $\vec{U}_{\epsilon}$ of the initial value problems

$$
\left\{\begin{array}{l}
\partial_{t} \vec{U}_{\epsilon}+S_{1}\left(\vec{U}_{\epsilon}\right) \partial_{x} \vec{U}_{\epsilon}+S_{2}\left(\vec{U}_{\epsilon}\right) \partial_{y} \vec{U}_{\epsilon}+\frac{1}{\epsilon} L \vec{U}_{\epsilon}=\vec{F}_{\epsilon} \\
\left.\vec{U}_{\epsilon}\right|_{t=0}=\vec{U}_{0, \epsilon}
\end{array}\right.
$$

exist (and are unique) in $C\left([0, T] ; \tilde{W}^{4}\right) \cap C^{1}\left([0, T] ; \tilde{W}^{3}\right)$ for all $\epsilon$, and are bounded in that space uniformly in $\epsilon$.

Theorem 2.5 implies Theorem 1.2 because uniform bounds on $u_{0 \epsilon}, v_{0 \epsilon}, h_{0 \epsilon}$ and $\vec{S}_{\epsilon}$ translate to uniform bounds on $\vec{U}_{0, \epsilon}$ and $\vec{F}_{\epsilon}$ after the changes of variables (2.1), (2.3), (2.6) and (2.7).

THEOREM 2.6 (convergence). Assume that the hypotheses of Theorem 2.5 are satisfied and let $\vec{U}_{\epsilon}$ be the solutions this theorem provides on $[0, T]$.

Assume, in addition, that $\vec{U}_{0, \epsilon}$ and $\vec{F}_{\epsilon}(t)$ converge respectively to $\vec{U}_{0}$ and $\vec{F}(t)$ in $L^{2}$, uniformly in $t$, and that $\left\|\vec{F}_{\epsilon}\right\|_{\operatorname{Lip}\left([0, T] ; L^{2}\right)} \leq C$.

Then

$$
\vec{U}_{\epsilon}=P_{0} \overrightarrow{\mathcal{U}}+\sum_{n=-1}^{\infty} e^{-i \frac{t}{\epsilon} \sqrt{2 n+3}} P_{1}^{(n)} \overrightarrow{\mathcal{U}}-\sum_{n=-1}^{\infty} e^{i \frac{t}{\epsilon} \sqrt{2 n+3}} P_{-1}^{(n)} \overrightarrow{\mathcal{U}}+o(1)
$$

in $C\left([0, T] ; \tilde{W}^{s}\right)$ for all $s<4$, where $\overrightarrow{\mathcal{U}} \in C\left([0, T] ; \tilde{W}^{4}\right) \cap C^{1}\left([0, T] ; \tilde{W}^{3}\right)$ satisfies the initial condition $\left.\overrightarrow{\mathcal{U}}\right|_{t=0}=\vec{U}_{0}$ and the equations

$$
\partial_{t} P_{0} \overrightarrow{\mathcal{U}}+P_{0} S_{1}^{0} \partial_{x} P_{0} \overrightarrow{\mathcal{U}}=P_{0} \vec{F}
$$

and, for all $n \geq 0$,

$$
\partial_{t} P_{ \pm 1}^{(n)} \overrightarrow{\mathcal{U}}+\frac{1}{2(2 n+3)} \partial_{x} P_{ \pm 1}^{(n)} \overrightarrow{\mathcal{U}}+c_{ \pm 1}^{(n)}\left(P_{0} \overrightarrow{\mathcal{U}}\right) P_{ \pm 1}^{(n)} \overrightarrow{\mathcal{U}}=0
$$

with

$$
c_{ \pm 1}^{(n)}\left(P_{0} \overrightarrow{\mathcal{U}}\right)=\left\langle S_{2}\left(P_{0} \overrightarrow{\mathcal{U}}\right) \partial_{y} \vec{e}_{ \pm 1}^{n}+S_{2}\left(\vec{e}_{ \pm 1}^{n}\right) \partial_{y} P_{0} \overrightarrow{\mathcal{U}}, \vec{e}_{ \pm 1}^{n}\right\rangle .
$$


The long-wave part of $\vec{U}_{\epsilon}$ is $P_{0} \vec{U}_{\epsilon}$; it converges strongly to $P_{0} \overrightarrow{\mathcal{U}}$. In the particular case of well-prepared initial data (that is, when $\left.P_{\mathrm{osc}} \vec{U}_{0}=0\right), \vec{U}_{\epsilon}$ itself converges strongly to $\overrightarrow{\mathcal{U}}$, which is then equal to $P_{0} \overrightarrow{\mathcal{U}}$, because $(2.26)$ and $\left.P_{ \pm 1}^{(n)} \overrightarrow{\mathcal{U}}\right|_{t=0}=0$ imply $P_{ \pm 1}^{(n)} \overrightarrow{\mathcal{U}} \equiv 0$ for all $n$.

To show that (2.25) is equivalent to the system (LW) of the beginning of Section 1 , let us set $\overrightarrow{\mathcal{V}}=P_{0} \overrightarrow{\mathcal{U}}$. Then $\overrightarrow{\mathcal{V}}$ is equal to

$$
\left(\begin{array}{l}
r \\
l \\
0
\end{array}\right)
$$

for some $r$ and $l$ such that

$$
L_{+} r+L_{-} l=0 \text {. }
$$

Since $P_{0} \vec{U}_{\epsilon} \rightarrow \overrightarrow{\mathcal{V}}$ is equivalent to $\tilde{P}_{0}\left(u_{\epsilon}, v_{\epsilon}, h_{\epsilon}\right) \rightarrow(u, 0, \tilde{h})$ with $(u, \tilde{h})$ related to $(r, l)$ by (2.4), we get the condition $y u+\partial_{y} h=0$ inside (LW), after dropping the . On the other hand, equation (2.25) may be written

$$
P_{0}\left(\partial_{t} \overrightarrow{\mathcal{V}}+S_{0}^{1} \partial_{x} \overrightarrow{\mathcal{V}}-\vec{F}\right)=0
$$

and for $\vec{U} \in\left(L^{2}\right)^{3}, P_{0} \vec{U}=0$ means that there exists some $v \in \tilde{W}^{1}$ such that

$$
U^{1}=L_{-} v, \quad U^{2}=L_{+} v .
$$

Indeed, ${ }^{t}\left(L_{-} v, L_{+} v, *\right)$ is orthogonal in $\left(L^{2}\right)^{3}$ to all vectors of the form $(2.27)$ satisfying (2.28) because $L_{+}$and $L_{-}$are adjoint to each other, and conversely, $P_{0} \vec{U}=0$ implies

$$
\begin{aligned}
\vec{U} & =\sum_{n=-1}^{+\infty} \sum_{\alpha=-1,1} U_{\alpha}^{(n)} \vec{e}_{\alpha}^{n} \\
& =\sum_{n=-1}^{+\infty} \sum_{\alpha=-1,1} \frac{U_{\alpha}^{(n)}}{\sqrt{2} \sqrt{2 n+3}}\left(\begin{array}{c}
\alpha i \sqrt{n+2} \phi_{n+2} \\
-\alpha i \sqrt{n+1} \phi_{n} \\
\sqrt{2 n+3} \phi_{n+1}
\end{array}\right) \\
& =\sum_{n=-1}^{+\infty} \sum_{\alpha=-1,1} \frac{U_{\alpha}^{(n)}}{\sqrt{2} \sqrt{2 n+3}}\left(\begin{array}{c}
-\alpha \frac{i}{\sqrt{2}} L_{-} \phi_{n+1} \\
-\alpha \frac{i}{\sqrt{2}} L_{+} \phi_{n+1} \\
\sqrt{2 n+3} \phi_{n+1}
\end{array}\right),
\end{aligned}
$$

hence (2.30) with

$$
v=-\sum_{n=-1}^{+\infty} \sum_{\alpha=-1,1} \frac{\alpha i U_{\alpha}^{(n)}}{2 \sqrt{2 n+3}} \phi_{n+1} .
$$

Therefore, (2.29) is equivalent to

$$
\left\{\begin{array}{l}
\partial_{t} r+\partial_{x} r+L_{-} v=\left(P_{0} \vec{F}\right)^{1} \\
\partial_{t} l-\partial_{x} l+L_{+} v=\left(P_{0} \vec{F}\right)^{2}
\end{array}\right.
$$

for some $v \in \tilde{W}^{1}$, which shows that the first two equations of the system (LW) are also satisfied. 


\section{Proofs}

3.1. Proof of existence-Main estimate. The existence of the solutions of (2.24) on a time interval of length independent of $\epsilon$ is proved as if the system was symmetric hyperbolic [15]: the initial data are mollified, and an iterative scheme brings back the problem to the linear case, which is in turn solved thanks to an a priori estimate on the linearized system.

So, what we have to prove here is an a priori estimate on the solution of (1.2) assuming $S=\left(S_{1}(\epsilon, \vec{V}), S_{2}(\vec{V})\right)$ with $\vec{V}$, as well as $\vec{F}$, continuous in time with values in $\tilde{W}^{4}$

To obtain the a priori estimates in $\tilde{W}^{4}$, according to Proposition 2.4, it is sufficient to estimate $\left\|H^{2} \vec{U}_{\epsilon}\right\|_{L^{2}}$ and $\left\|\partial_{x}^{4} \vec{U}_{\epsilon}\right\|_{L^{2}}$. With only the $y$-dependence of the singular terms, the estimates for $\left\|\partial_{x}^{4} \vec{U}_{\epsilon}\right\|_{L^{2}}$ are straightforward. Below we emphasize the new estimate for $\left\|H^{2} \vec{U}_{\epsilon}\right\|_{L^{2}}$.

Developing the terms in $\epsilon^{-1}$ in the system using the parabolic cylinder functions gives

$$
\begin{aligned}
& \partial_{t} r+\underline{S} \cdot \nabla r-\frac{1}{\epsilon} v_{0} \phi_{1}-\frac{1}{\epsilon} \sum_{n=0}^{\infty} v_{n+1}(n+2)^{1 / 2} \phi_{n+2}=F^{1} \\
& \partial_{t} l+\underline{S} \cdot \nabla l+\frac{1}{\epsilon} \sum_{n=0}^{\infty} v_{n+1}(n+1)^{1 / 2} \phi_{n}=F^{2} \\
& \partial_{t} v+\underline{S} \cdot \nabla v+\frac{1}{\epsilon} r_{1} \phi_{0}+\frac{1}{\epsilon} \sum_{n=0}^{\infty}\left(r_{n+2}(n+2)^{1 / 2}-l_{n}(n+1)^{1 / 2}\right) \phi_{n+1}=F^{3} .
\end{aligned}
$$

Define $H_{r}$ and $H_{v}$ by

$$
\begin{aligned}
& H_{r} f=f_{0} \phi_{0}+f_{1} \phi_{1}-\sum_{n=0}^{\infty} f_{n+2}(2 n+1) \phi_{n+2} \\
& H_{v} f=f_{0} \phi_{0}-\sum_{n=0}^{\infty} f_{n+1}(2 n+1) \phi_{n+1} .
\end{aligned}
$$

If we apply $H_{r}^{2}$ to $(3.1)$ and then take the scalar product with $H_{r}^{2} r$ in $L^{2}$, we get

$$
\begin{aligned}
\frac{1}{2} \partial_{t}\left\|H_{r}^{2} r\right\|_{L^{2}}^{2} & +\iint H_{r}^{2} r H_{r}^{2}(\underline{S} \cdot \nabla r) d x d y \\
+ & \frac{1}{\epsilon} \int\left(-r_{1} v_{0}-\sum_{n=0}^{\infty} r_{n+2} v_{n+1}(n+2)^{1 / 2}(2 n+1)^{4}\right) d x \\
& =\iint H_{r}^{2} r H_{r}^{2} F^{1} d x d y .
\end{aligned}
$$

Similarly, applying $H^{2}$ to (3.2) and $H_{v}^{2}$ to (3.3), we get

$$
\begin{aligned}
\frac{1}{2} \partial_{t}\left\|H^{2} l\right\|_{L^{2}}^{2} & +\iint H^{2} l H^{2}(\underline{S} \cdot \nabla l) d x d y \\
+ & \frac{1}{\epsilon} \int \sum_{n=0}^{\infty} l_{n} v_{n+1}(n+1)^{1 / 2}(2 n+1)^{4} d x \\
& =\iint H^{2} l H^{2} F^{2} d x d y
\end{aligned}
$$


and

$$
\begin{aligned}
\frac{1}{2} \partial_{t}\left\|H_{v}^{2} v\right\|_{L^{2}}^{2} & +\iint H_{v}^{2} v H_{v}^{2}(\underline{S} \cdot \nabla v) d x d y \\
+ & \frac{1}{\epsilon} \int\left(r_{1} v_{0}+\sum_{n=0}^{\infty} v_{n+1}\left(r_{n+2}(n+2)^{1 / 2}-l_{n}(n+1)^{1 / 2}\right)(2 n+1)^{4}\right) d x \\
& =\iint H_{v}^{2} v H_{v}^{2} F^{3} d x d y .
\end{aligned}
$$

Additioning (3.4), (3.5) and (3.6), the terms in $\epsilon^{-1}$ vanish:

$$
\begin{aligned}
& \frac{1}{2} \partial_{t}\left(\left\|H_{r}^{2} r\right\|_{L^{2}}^{2}+\left\|H^{2} l\right\|_{L^{2}}^{2}+\left\|H_{v}^{2} v\right\|_{L^{2}}^{2}\right) \\
& \quad+\iint H_{r}^{2}(\underline{S} \cdot \nabla r) H_{r}^{2} r d x d y \\
& \quad+\iint H^{2}(\underline{S} \cdot \nabla l) H^{2} l d x d y \\
& \quad+\iint H_{v}^{2}(\underline{S} \cdot \nabla v) H_{v}^{2} v d x d y \\
& \quad=\iint\left(H_{r}^{2} F^{1} H_{r}^{2} r+H^{2} F^{2} H^{2} l+H_{v}^{2} F^{3} H_{v}^{2} v\right) d x d y .
\end{aligned}
$$

The sum of (3.7), (3.8) and (3.9) is equal to

$$
\begin{aligned}
& \left.\iint\left(\underline{S} \cdot \nabla H^{2} \vec{U}\right) \cdot H^{2} \vec{U}\right) d x d y \\
+ & \iint\left(\left[H^{2}, \underline{S} \cdot \nabla\right] \vec{U}\right) \cdot H^{2} \vec{U} d x d y \\
+ & \iint\left(H_{r}^{2}-H^{2}\right)(\underline{S} \cdot \nabla r) H_{r}^{2} r d x d y \\
+ & \iint H^{2}(\underline{S} \cdot \nabla r)\left(H_{r}^{2}-H^{2}\right) r d x d y \\
+ & \iint\left(H_{v}^{2}-H^{2}\right)(\underline{S} \cdot \nabla v) H_{v}^{2} v d x d y \\
+ & \iint H^{2}(\underline{S} \cdot \nabla v)\left(H_{v}^{2}-H^{2}\right) v d x d y .
\end{aligned}
$$

In the first term, the $\nabla$ can be put on $\underline{S}$ by integration by parts, thanks to the symmetry of $\underline{S}$ :

$$
\begin{aligned}
& \iint\left.\left(\underline{S} \cdot \nabla H^{2} \vec{U}\right) \cdot H^{2} \vec{U}\right) d x d y \\
& \quad=\iint \sum_{i, j=1}^{3}\left(\underline{S}^{i j} \cdot \nabla H^{2} U^{j}\right) H^{2} U^{i} d x d y \\
& \quad=\iint \frac{1}{2} \sum_{i, j=1}^{3} \underline{S}^{i j} \cdot \nabla\left(H^{2} U^{i} H^{2} U^{j}\right) d x d y \\
& \quad=-\iint \frac{1}{2} \sum_{i, j=1}^{3}\left(\partial_{x} S_{1}^{i j}+\partial_{y} S_{2}^{i j}\right) H^{2} U^{i} H^{2} U^{j} d x d y,
\end{aligned}
$$


hence

$$
|(3.11)| \leq C\left(\epsilon\left\|\partial_{x} \vec{V}\right\|_{L^{\infty}}+\left\|\partial_{y} \vec{V}\right\|_{L^{\infty}}\right)\left\|H^{2} \vec{U}\right\|_{L^{2}}^{2}
$$

The commutator in (3.12) is expanded explicitly and each term is estimated in $L^{2}$; as

$$
H^{2}=\left(\partial_{y}^{2}-y^{2}\right)\left(\partial_{y}^{2}-y^{2}\right)=\partial_{y}^{4}-2 y^{2} \partial_{y}^{2}-4 y \partial_{y}+y^{4}-2,
$$

we just have four commutators to estimate.

- With $\partial_{y}^{4}$ :

$$
\begin{aligned}
\left\|\left[\partial_{y}^{4}, \underline{S} \cdot \nabla\right] \vec{U}\right\|_{L^{2}}= & \left\|\left[\partial_{y}^{4}, \underline{S}\right] \cdot \nabla \vec{U}\right\|_{L^{2}} \\
\leq & \left\|\left[\partial_{y}^{4}, S_{1}\right] \partial_{x} \vec{U}\right\|_{L^{2}}+\left\|\left[\partial_{y}^{4}, S_{2}\right] \partial_{y} \vec{U}\right\|_{L^{2}} \\
\leq & C\left(\left\|\partial_{y} S_{1}\right\|_{L^{\infty}}\|\vec{U}\|_{W^{4}}+\epsilon\|\vec{V}\|_{W^{4}}\left\|\partial_{x} \vec{U}\right\|_{L^{\infty}}\right) \\
& +C\left(\left\|\partial_{y} S_{2}\right\|_{L^{\infty}}\|\vec{U}\|_{W^{4}}+\left\|S_{2}\right\|_{W^{4}}\left\|\partial_{y} \vec{U}\right\|_{L^{\infty}}\right) \\
\leq & C\left(\epsilon\left\|\partial_{x} \vec{U}\right\|_{L^{\infty}}+\left\|\partial_{y} \vec{U}\right\|_{L^{\infty}}\right)\|\vec{V}\|_{W^{4}} \\
& +C\left\|\partial_{y} \vec{V}\right\|_{L^{\infty}}\|\vec{U}\|_{W^{4}}
\end{aligned}
$$

thanks to the classical estimates of such commutators in Sobolev spaces (here and below we may replace $\left\|S_{1}\right\|$ by $\epsilon\|\vec{V}\|$ whatever \|\| is, because the constant part of $S_{1}$ commutes).

- With $y^{2} \partial_{y}^{2}$ :

$$
\begin{aligned}
\left\|\left[y^{2} \partial_{y}^{2}, \underline{S} \cdot \nabla\right] \vec{U}\right\|_{L^{2}} \leq & \left\|y^{2} \partial_{y}^{2} S_{1} \partial_{x} \vec{U}\right\|_{L^{2}}+\left\|2 y^{2} \partial_{y} S_{1} \partial_{x} \partial_{y} \vec{U}\right\|_{L^{2}} \\
& +\left\|y^{2} \partial_{y}^{2} S_{2} \partial_{y} \vec{U}\right\|_{L^{2}}+\left\|2 y^{2} \partial_{y} S_{2} \partial_{y}^{2} \vec{U}\right\|_{L^{2}} \\
& +\left\|2 y S_{2} \partial_{y}^{2} \vec{U}\right\|_{L^{2}} \\
\leq & \left\|y^{2} \partial_{y}^{2} S_{1}\right\|_{L^{2}}\left\|\partial_{x} \vec{U}\right\|_{L^{\infty}}+2\left\|\partial_{y} S_{1}\right\|_{L^{\infty}}\left\|y^{2} \partial_{x} \partial_{y} \vec{U}\right\|_{L^{2}} \\
& +\left\|y^{2} \partial_{y}^{2} S_{2}\right\|_{L^{2}}\left\|\partial_{y} \vec{U}\right\|_{L^{\infty}}+2\left\|\partial_{y} S_{2}\right\|_{L^{\infty}}\left\|y^{2} \partial_{y}^{2} \vec{U}\right\|_{L^{2}} \\
& +2\left\|S_{2}\right\|_{L^{\infty}}\left\|y \partial_{y} \vec{U}\right\|_{L^{2}} \\
\leq & 2\left(\left\|S_{2}\right\|_{L^{\infty}}+\left\|\partial_{y} S_{2}\right\|_{L^{\infty}}\right)\|\vec{U}\|_{\tilde{W}^{4}}+\left\|\partial_{y} \vec{U}\right\|_{L^{\infty}}\left\|S_{2}\right\|_{\tilde{W}^{4}} \\
& +\left\|\partial_{x} \vec{U}\right\|_{L^{\infty}}\left\|S_{1}\right\|_{\tilde{W}^{4}} \\
\leq & C\left(\|\vec{V}\|_{L^{\infty}}+\left\|\partial_{y} \vec{V}\right\|_{L^{\infty}}\right)\|\vec{U}\|_{\tilde{W}^{4}} \\
& +C\left(\epsilon\left\|\partial_{x} \vec{U}\right\|_{L^{\infty}}+\left\|\partial_{y} \vec{U}\right\|_{L^{\infty}}\right)\|\vec{V}\|_{\tilde{W}^{4}}
\end{aligned}
$$

- With $y \partial_{y}$ :

$$
\begin{aligned}
\left\|\left[y \partial_{y}, \underline{S} \cdot \nabla\right] \vec{U}\right\|_{L^{2}} & \leq\left\|y \partial_{y} S_{1} \partial_{x} \vec{U}\right\|_{L^{2}}+\left\|y \partial_{y} S_{2} \partial_{y} \vec{U}\right\|_{L^{2}}+\left\|S_{2} \partial_{y} \vec{U}\right\|_{L^{2}} \\
& \leq\left\|\partial_{x} \vec{U}\right\|_{L^{\infty}}\left\|S_{1}\right\|_{\tilde{W}^{2}}+2\left\|\partial_{y} \vec{U}\right\|_{L^{\infty}}\left\|S_{2}\right\|_{\tilde{W}^{2}} \\
& \leq C\left(\epsilon\left\|\partial_{x} \vec{U}\right\|_{L^{\infty}}+\left\|\partial_{y} \vec{U}\right\|_{L^{\infty}}\right)\|\vec{V}\|_{\tilde{W}^{2}} .
\end{aligned}
$$

- With $y^{4}$ :

$$
\begin{aligned}
\left\|\left[y^{4}, \underline{S} \cdot \nabla\right] \vec{U}\right\|_{L^{2}} & =\left\|4 S_{2} y^{3} \vec{U}\right\|_{L^{2}} \\
& \leq 4\left\|S_{2}\right\|_{L^{\infty}}\|\vec{U}\|_{\tilde{W}^{3}} \\
& \leq C\|\vec{V}\|_{L^{\infty}}\|\vec{U}\|_{\tilde{W}^{3}} .
\end{aligned}
$$


So

$$
\begin{aligned}
|(3.12)| \leq & C\left(\|\vec{V}\|_{L^{\infty}}+\left\|\partial_{y} V\right\|_{L^{\infty}}+\epsilon\left\|\partial_{x} \vec{V}\right\|_{L^{\infty}}\right)\|\vec{U}\|_{\tilde{W}^{4}}^{2} \\
& +C\left(\left\|\partial_{y} \vec{U}\right\|_{L^{\infty}}+\epsilon\left\|\partial_{x} \vec{U}\right\|_{L^{\infty}}\right)\|\vec{V}\|_{\tilde{W}^{4}}\|\vec{U}\|_{\tilde{W}^{4}} .
\end{aligned}
$$

The terms (3.13-3.16) are easy to estimate because the difference between $H_{r}^{2}$ (or $H_{v}^{2}$ ) and $H^{2}$ contains derivatives and multiplications in $y$ only up to order two. Indeed:

$$
\begin{aligned}
\left(H_{r}^{2}\right. & \left.-H^{2}\right)\left(\sum_{n=0}^{\infty} f_{n} \phi_{n}\right) \\
& =f_{0} \phi_{0}+f_{1} \phi_{1}+\sum_{n=0}^{\infty} f_{n+2}(2 n+1)^{2} \phi_{n+2}-\sum_{n=0}^{\infty} f_{n}(2 n+1)^{2} \phi_{n} \\
& =-8 f_{1} \phi_{1}-8 \sum_{n=0}^{\infty} f_{n+2}(2 n+5-2) \phi_{n+2} \\
& =-8 f_{1} \phi_{1}+16\left(f-f_{0} \phi_{0}-f_{1} \phi_{1}\right)-8\left(H f-f_{0} \phi_{0}-3 \phi_{1}\right) \\
& =-8 H f+16 f-8 f_{0} \phi_{0} .
\end{aligned}
$$

Therefore

$$
\left\|\left(H_{r}^{2}-H^{2}\right)(\underline{S} \cdot \nabla r)\right\|_{L^{2}} \leq C\|\underline{S} \cdot \nabla r\|_{L^{2}}+C\|H(\underline{S} \cdot \nabla r)\|_{L^{2}},
$$

and since

$$
\begin{aligned}
\|H(\underline{S} \cdot \nabla r)\|_{L^{2}}= & \left\|\partial_{y}^{2}(\underline{S} \cdot \nabla r)-y^{2}(\underline{S} \cdot \nabla r)\right\|_{L^{2}} \\
\leq & \left\|\left(\partial_{y}^{2} \underline{S}\right) \cdot \nabla r\right\|_{L^{2}}+\left\|2\left(\partial_{y} \underline{S}\right) \cdot \nabla \partial_{y} r\right\|_{L^{2}}+\left\|\underline{S} \cdot \nabla \partial_{y}^{2} r\right\|_{L^{2}} \\
& +\left\|y^{2} \underline{S} \cdot \nabla r\right\|_{L^{2}} \\
\leq & C\left(\left\|\partial_{y} \vec{U}\right\|_{L^{\infty}}+\epsilon\left\|\partial_{x} \vec{U}\right\|_{L^{\infty}}\right)\|\vec{V}\|_{W^{2}} \\
& +C\left(\|\vec{V}\|_{L^{\infty}}+\left\|\partial_{y} \vec{V}\right\|_{L^{\infty}}+\epsilon\left\|\partial_{x} \vec{V}\right\|_{L^{\infty}}\right)\|\vec{U}\|_{\tilde{W}^{3}},
\end{aligned}
$$

we have

$$
\begin{aligned}
|(3.13)| \leq & C\left(\left\|\partial_{y} \vec{U}\right\|_{L^{\infty}}+\epsilon\left\|\partial_{x} \vec{U}\right\|_{L^{\infty}}\right)\|\vec{V}\|_{W^{2}}\left\|H_{r}^{2} r\right\|_{L^{2}} \\
& +C\left(\|\vec{V}\|_{L^{\infty}}+\left\|\partial_{y} \vec{V}\right\|_{L^{\infty}}+\epsilon\left\|\partial_{x} \vec{V}\right\|_{L^{\infty}}\right)\|\vec{U}\|_{\tilde{W}^{3}}\left\|H_{r}^{2} r\right\|_{L^{2}} .
\end{aligned}
$$

Similarly,

$$
\begin{aligned}
|(3.15)| \leq & C\left(\left\|\partial_{y} \vec{U}\right\|_{L^{\infty}}+\epsilon\left\|\partial_{x} \vec{U}\right\|_{L^{\infty}}\right)\|\vec{V}\|_{W^{2}}\left\|H_{v}^{2} v\right\|_{L^{2}} \\
& +C\left(\|\vec{V}\|_{L^{\infty}}+\left\|\partial_{y} \vec{V}\right\|_{L^{\infty}}+\epsilon\left\|\partial_{x} \vec{V}\right\|_{L^{\infty}}\right)\|\vec{U}\|_{\tilde{W}^{3}}\left\|H_{v}^{2} v\right\|_{L^{2}} .
\end{aligned}
$$

Because $H$ is self-adjoint, we may write

$$
\begin{aligned}
(3.14) & =\iint H(\underline{S} \cdot \nabla r) H\left(H_{r}^{2}-H^{2}\right) r d x d y \\
& =\iint H(\underline{S} \cdot \nabla r)\left(-8 H^{2} r+16 H r-8 f_{0} \phi_{0}\right) d x d y .
\end{aligned}
$$

Then we use the Cauchy-Schwarz inequality and the estimate (3.19), which gives

$$
\begin{aligned}
|(3.14)| \leq & C\left(\left\|\partial_{y} \vec{U}\right\|_{L^{\infty}}+\epsilon\left\|\partial_{x} \vec{U}\right\|_{L^{\infty}}\right)\|\vec{V}\|_{W^{2}}\|r\|_{\tilde{W}^{4}} \\
& +C\left(\|\vec{V}\|_{L^{\infty}}+\left\|\partial_{y} \vec{V}\right\|_{L^{\infty}}+\epsilon\left\|\partial_{x} \vec{V}\right\|_{L^{\infty}}\right)\|\vec{U}\|_{\tilde{W}^{3}}\|r\|_{\tilde{W}^{4}} .
\end{aligned}
$$


Similarly,

$$
\begin{aligned}
|(3.16)| \leq & C\left(\left\|\partial_{y} \vec{U}\right\|_{L^{\infty}}+\epsilon\left\|\partial_{x} \vec{U}\right\|_{L^{\infty}}\right)\|\vec{V}\|_{W^{2}}\|v\|_{\tilde{W}^{4}} \\
& +C\left(\|\vec{V}\|_{L^{\infty}}+\left\|\partial_{y} \vec{V}\right\|_{L^{\infty}}+\epsilon\left\|\partial_{x} \vec{V}\right\|_{L^{\infty}}\right)\|\vec{U}\|_{\tilde{W}^{3}}\|v\|_{\tilde{W}^{4}} .
\end{aligned}
$$

Summing up, we have

$$
\begin{aligned}
& \left\|H^{2} \vec{U}(t)\right\|_{L^{2}} \\
\leq & C\left(\left\|H_{r}^{2} \vec{U}(t)\right\|_{L^{2}}+\left\|H^{2} l(t)\right\|_{L^{2}}+\left\|H_{v}^{2} v(t)\right\|_{L^{2}}\right) \\
\leq & C\|\vec{U}(0)\|_{\tilde{W}^{4}}+C \int_{0}^{t}\left\|F\left(t^{\prime}\right)\right\|_{\tilde{W}^{4}} d t^{\prime} \\
+ & C \int_{0}^{t}\left(\left\|\vec{V}\left(t^{\prime}\right)\right\|_{L^{\infty}}+\left\|\partial_{y} \vec{V}\left(t^{\prime}\right)\right\|_{L^{\infty}}+\epsilon\left\|\partial_{x} \vec{V}\left(t^{\prime}\right)\right\|_{L^{\infty}}\right)\left\|\vec{U}\left(t^{\prime}\right)\right\|_{\tilde{W}^{4}} d t^{\prime} \\
+ & C \int_{0}^{t}\left(\left\|\partial_{y} \vec{U}\left(t^{\prime}\right)\right\|_{L^{\infty}}+\epsilon\left\|\partial_{x} \vec{U}\left(t^{\prime}\right)\right\|_{L^{\infty}}\right)\left\|\vec{V}\left(t^{\prime}\right)\right\|_{\tilde{W}^{4}} d t^{\prime} .
\end{aligned}
$$

By a proof of the same kind, only simpler, this last expression also bounds $\left\|\partial_{x}^{4} \vec{U}(t)\right\|_{L^{2}}$, and therefore $\|\vec{U}(t)\|_{\tilde{W}^{4}}$.

3.2. Fast averaging. We apply the method of fast averaging introduced by Schochet [25].

Using the projectors of Section 2.5, we define for any $\tau \in \mathbb{R}$ the exponential of $\tau L$ by

$$
e^{\tau L} \vec{U}=\sum_{n=-1}^{+\infty} \sum_{\alpha=-1}^{+1} e^{\alpha i \tau \sqrt{2 n+3}} P_{\alpha}^{(n)} \vec{U}
$$

for all $\vec{U} \in\left(L^{2}\right)^{3}$.

Proposition 3.1. There is a constant $C$ such that $\left\|e^{\tau L} \vec{U}\right\|_{\tilde{W}^{s}} \leq C\|\vec{U}\|_{\tilde{W}^{s}}$ for any $\tau \in \mathbb{R}$, any $s \geq 0$ and any $\vec{U} \in \tilde{W}^{s}$.

Proof. The coefficients of $\left(e^{\tau L} \vec{U}\right)^{1}$ in the decomposition (2.19) are

$$
\begin{aligned}
& \left(e^{\tau L} \vec{U}\right)_{k(n+2)}^{1} \\
& =\left(\sum_{\alpha=-1}^{+1} e^{\alpha i \tau \sqrt{2 n+3}} P_{\alpha}^{(n)} \vec{U}\right)_{k(n+2)}^{1} \\
& =\left(\sum_{\alpha=-1}^{+1} e^{\alpha i \tau \sqrt{2 n+3}}\left(\int_{\mathbb{R}} \vec{U}\left(x, y^{\prime}\right) \cdot \overline{\vec{e}_{\alpha}^{n}\left(y^{\prime}\right)} d y^{\prime}\right)\left(\vec{e}_{\alpha}^{n}\right)^{1}(y)\right)_{k(n+2)} \\
& =\sum_{\alpha=-1}^{+1} e^{\alpha i \tau \sqrt{2 n+3}} \int_{\mathbb{R}}\left(\vec{e}_{\alpha}^{n}\right)^{1}(y) \phi_{n+2}(y) d y \int_{\mathbb{T}}\left(\int_{\mathbb{R}} \vec{U}\left(x, y^{\prime}\right) \cdot \overline{\vec{e}_{\alpha}^{n}\left(y^{\prime}\right)} d y^{\prime}\right) e^{-2 \pi i k x} d x,
\end{aligned}
$$

so

$$
\left|\left(e^{\tau L} \vec{U}\right)_{k(n+2)}^{1}\right| \leq C\left(\left|U_{k(n+2)}^{1}\right|+\left|U_{k n}^{2}\right|+\left|U_{k(n+1)}^{3}\right|\right) .
$$

The same calculation shows that the coefficients $\left(e^{\tau L} \vec{U}\right)_{k n}^{2}$ and $\left(e^{\tau L} \vec{U}\right)_{k(n+1)}^{3}$ are also bounded by (3.25). 
LemMA 3.2. Let $s \geq 0$. If $\vec{U} \in C\left([0, T] ; \tilde{W}^{s}\right)$, the series

$$
\sum_{n=-1}^{+\infty} \sum_{\alpha=-1}^{+1} e^{\alpha i \frac{t}{\epsilon} \sqrt{2 n+3}} P_{\alpha}^{(n)} \vec{U}(t)
$$

converges to $e^{\frac{t}{\epsilon} L} \vec{U}(t)$ in $C\left([0, T] ; \tilde{W}^{s}\right)$.

Proof. For any $\eta>0$, there exists $N>0$ such that

$$
\left\|\vec{U}(t)-\sum_{n=-1}^{N} \sum_{\alpha=-1}^{+1} P_{\alpha}^{(n)} \vec{U}(t)\right\|_{\tilde{W}^{s}} \leq \eta
$$

for all $t \in[0, T]$, because $\vec{U}$ is uniformly continuous on $[0, T]$, and

$$
\begin{aligned}
\| e^{\frac{t}{\epsilon} L} \vec{U}(t) & -\sum_{n=-1}^{N} \sum_{\alpha=-1}^{+1} e^{\alpha i \frac{t}{\epsilon} \sqrt{2 n+3}} P_{\alpha}^{(n)} \vec{U}(t) \|_{\tilde{W}^{s}} \\
& =\left\|e^{\frac{t}{\epsilon} L}\left(\vec{U}(t)-\sum_{n=-1}^{N} \sum_{\alpha=-1}^{+1} P_{\alpha}^{(n)} \vec{U}(t)\right)\right\|_{\tilde{W}^{s}} \\
& =\left\|\vec{U}(t)-\sum_{n=-1}^{N} \sum_{\alpha=-1}^{+1} P_{\alpha}^{(n)} \vec{U}(t)\right\|_{\tilde{W}^{s}}
\end{aligned}
$$

by Proposition 3.1.

LEMMA 3.3. Let $s \geq 0$. If $\vec{U} \in C\left([0, T] ; \tilde{W}^{s}\right)$, the series

$$
\sum_{n=-1}^{+\infty} \sum_{\alpha=-1}^{+1} e^{\alpha i \tau \sqrt{2 n+3}} P_{\alpha}^{(n)} \vec{U}(t)
$$

converges to $e^{\tau L} \vec{U}(t)$ in $C\left([0, T] ; \tilde{W}^{s}\right)$, uniformly with respect to $\tau \in \mathbb{R}$.

Proof. Same as above.

Proposition 3.4. If $\vec{U} \in C^{1}\left([0, T] ; L^{2}\right) \cap C\left([0, T] ; \tilde{W}^{1}\right)$, then

$$
\partial_{t}\left(e^{\frac{t}{\epsilon} L} \vec{U}\right)=e^{\frac{t}{\epsilon} L}\left(\partial_{t} \vec{U}+\frac{1}{\epsilon} L \vec{U}\right)
$$

Proof. The derivation of (3.26) term by term gives

$$
\sum_{n=-1}^{+\infty} \sum_{\alpha=-1}^{+1} e^{\alpha i \frac{t}{\epsilon} \sqrt{2 n+3}}\left(P_{\alpha}^{(n)} \partial_{t} \vec{U}(t)+\frac{1}{\epsilon} \alpha i \sqrt{2 n+3} P_{\alpha}^{(n)} \vec{U}(t)\right) .
$$

Since

$$
\sum_{n=-1}^{+\infty} \sum_{\alpha=-1}^{+1} \alpha i \sqrt{2 n+3} P_{\alpha}^{(n)} \vec{U}(t)=\sum_{n=-1}^{+\infty} \sum_{\alpha=-1}^{+1} P_{\alpha}^{(n)}(L \vec{U}(t))
$$

the series $(3.28)$ converges to $e^{\frac{t}{\epsilon} L}\left(\partial_{t} \vec{U}+\frac{1}{\epsilon} L \vec{U}\right)$ in $C\left([0, T] ; L^{2}\right)$, by Lemma 3.2, which justifies the derivation term by term. 
By Proposition 3.4, the solutions of (2.24) satisfy

$$
\partial_{t}\left(e^{\frac{t}{\epsilon} L} \vec{U}_{\epsilon}\right)=e^{\frac{t}{\epsilon} L}\left(\vec{F}_{\epsilon}-\underline{S}\left(\epsilon, \vec{U}_{\epsilon}\right) \cdot \nabla \vec{U}_{\epsilon}\right)
$$

and therefore $\partial_{t}\left(e^{\frac{t}{\epsilon} L} \vec{U}_{\epsilon}\right)$ is bounded in $C\left([0, T] ; \tilde{W}^{3}\right)$, uniformly in $\epsilon$. We apply the following version of the Lions-Aubin compactness lemma with $s=4$ and $s^{\prime}=3$.

LEMMA 3.5. Let $0 \leq s^{\prime}<s$. If functions $f_{\epsilon}$ are bounded in $C\left([0, T] ; \tilde{W}^{s}\right) \cap$ $\operatorname{Lip}\left([0, T] ; \tilde{W}^{s^{\prime}}\right)$, uniformly in $\epsilon$, there is a sequence $\left(f_{\epsilon(n)}\right)$, with $\epsilon(n) \searrow 0$, that converges in $C\left([0, T] ; \tilde{W}^{s^{\prime \prime}}\right)$ for all $s^{\prime \prime}<s$, and the limit belongs to $\operatorname{Lip}\left([0, T] ; \tilde{W}^{s^{\prime}}\right)$.

Thus we get

$$
e^{\frac{t}{\epsilon} L} \vec{U}_{\epsilon} \rightarrow \overrightarrow{\mathcal{U}} \quad \text { in } C\left([0, T] ; \tilde{W}^{3}\right) \subset C\left([0, T] ; L^{2} \cap \operatorname{Lip}\right)
$$

as $\epsilon=\epsilon(n) \searrow 0$ (eventually it will turn out that the limit $\overrightarrow{\mathcal{U}}$ is unique, so there really is no need to extract), and

$$
\overrightarrow{\mathcal{U}} \in \operatorname{Lip}\left([0, T] ; \tilde{W}^{3}\right) \subset \operatorname{Lip}\left([0, T] ; L^{2} \cap \operatorname{Lip}\right) .
$$

The equations satisfied by $\overrightarrow{\mathcal{U}}$ are obtained as follows. Integrating (3.29) gives

$$
e^{\frac{t}{\epsilon} L} \vec{U}_{\epsilon}(t)=\vec{U}_{0, \epsilon}+\int_{0}^{t} e^{\frac{t^{\prime}}{\epsilon} L}\left(\vec{F}_{\epsilon}\left(t^{\prime}\right)-\underline{S}\left(\epsilon, \vec{U}_{\epsilon}\left(t^{\prime}\right)\right) \cdot \nabla \vec{U}_{\epsilon}\left(t^{\prime}\right)\right) d t^{\prime} .
$$

The left-hand side of (3.32) converges to $\overrightarrow{\mathcal{U}}$ in $C\left([0, T] ; L^{2}\right)$. By assumption, $\vec{U}_{0, \epsilon} \rightarrow \vec{U}_{0}$ in $L^{2}$ and $\vec{F}_{\epsilon} \rightarrow \vec{F}$ in $C\left([0, T] ; L^{2}\right)$. Since $\vec{U}_{\epsilon}-e^{-\dot{\epsilon} L} \overrightarrow{\mathcal{U}} \rightarrow 0$ in $C\left([0, T] ; L^{2} \cap \operatorname{Lip}\right)$, by (3.30) and Lemma 3.1, $\underline{S}\left(\epsilon, \vec{U}_{\epsilon}\right) \cdot \nabla \vec{U}_{\epsilon}-\underline{S}\left(0, e^{\dot{\bar{\epsilon}} L} \overrightarrow{\mathcal{U}}\right) \cdot \nabla\left(e^{-\dot{\bar{\epsilon}} L} \overrightarrow{\mathcal{U}}\right) \rightarrow 0$ in $C\left([0, T] ; L^{2}\right)$. Therefore,

$$
\overrightarrow{\mathcal{U}}(t)=\vec{U}_{0}+\left.\lim _{\epsilon \rightarrow 0} \int_{0}^{t} h\left(t^{\prime}, \tau\right)\right|_{\tau=\frac{t^{\prime}}{\epsilon}} d t^{\prime}
$$

with

$$
\begin{aligned}
h(t, \tau) & =e^{\tau L}\left(\vec{F}(t)-\underline{S}\left(0, e^{-\tau L} \overrightarrow{\mathcal{U}}(t)\right) \cdot \nabla\left(e^{-\tau L} \overrightarrow{\mathcal{U}}(t)\right)\right) \\
& =e^{\tau L}\left(\vec{F}(t)-S_{1}^{0}\left(e^{-\tau L} \partial_{x} \overrightarrow{\mathcal{U}}(t)\right)-S_{2}\left(e^{-\tau L} \overrightarrow{\mathcal{U}}(t)\right) \partial_{y}\left(e^{-\tau L} \overrightarrow{\mathcal{U}}(t)\right) .\right.
\end{aligned}
$$

The limit in (3.33) can be evaluated thanks to a lemma by Schochet [26].

Lemma 3.6. Let $h \in C\left([0, T] \times \mathbb{R} ; L^{2}\right)$. Assume that $\left\|h\left(t_{1}, \tau\right)-h\left(t_{2}, \tau\right)\right\|_{L^{2}} \leq C\left|t_{1}-t_{2}\right|$, uniformly in $\tau$, and that

$$
\frac{1}{T_{1}} \int_{T_{0}}^{T_{0}+T_{1}} h(t, \tau) d \tau \rightarrow[M h](t)
$$

in $C\left([0, T] ; L^{2}\right)$, uniformly in $T_{0}$, as $T_{1} \rightarrow+\infty$. Then

$$
\int_{0}^{t} h\left(t^{\prime}, \frac{t^{\prime}}{\epsilon}\right) d t^{\prime} \rightarrow \int_{0}^{t}[M h]\left(t^{\prime}\right) d t^{\prime}
$$

in $C\left([0, T] ; L^{2}\right)$ as $\epsilon \rightarrow 0$.

Proof. See [26, Lemma 3.2]. 
Let us compute $M h$ when $h$ given by (3.34). As

$$
e^{\tau L} \vec{F}-e^{\tau L} \sum_{n=-1}^{N} \sum_{\alpha=-1}^{+1} P_{\alpha}^{(n)} \vec{F} \rightarrow 0
$$

in $C\left([0, T] \times \mathbb{R} ; L^{2}\right)$, by Lemma 3.3 ,

$$
\frac{1}{T_{1}} \int_{T_{0}}^{T_{0}+T_{1}} e^{\tau L} \vec{F} d \tau-\frac{1}{T_{1}} \int_{T_{0}}^{T_{0}+T_{1}} e^{\tau L} \sum_{n=-1}^{N} \sum_{\alpha=-1}^{+1} P_{\alpha}^{(n)} \vec{F} d \tau
$$

converges to zero in $C\left([0, T] ; L^{2}\right)$, uniformly in $T_{0}$ and $T_{1}$. For each $n$ and $\alpha$,

$$
\begin{aligned}
\frac{1}{T_{1}} \int_{T_{0}}^{T_{0}+T_{1}} e^{\tau L} P_{\alpha}^{(n)} \vec{F} d \tau & =\frac{1}{T_{1}} \int_{T_{0}}^{T_{0}+T_{1}} e^{\alpha i \tau \sqrt{2 n+3}} d \tau P_{\alpha}^{(n)} \vec{F} \\
& \rightarrow \begin{cases}P_{\alpha}^{(n)} \vec{F} & \text { if } \alpha=0, \\
0 & \text { if } \alpha= \pm 1,\end{cases}
\end{aligned}
$$

as $T_{1} \rightarrow \infty$, uniformly in $T_{0}$. So

$$
\lim _{T_{1} \rightarrow \infty} \frac{1}{T_{1}} \int_{T_{0}}^{T_{0}+T_{1}} e^{\tau L} \vec{F} d \tau=\sum_{n=-1}^{+\infty} P_{0}^{(n)} \vec{F}=P_{0} \vec{F} .
$$

In the same way,

$$
\begin{aligned}
\lim _{T_{1} \rightarrow \infty} & \frac{1}{T_{1}} \int_{T_{0}}^{T_{0}+T_{1}} e^{\tau L} S_{1}^{0}\left(e^{-\tau L} \partial_{x} \overrightarrow{\mathcal{U}}\right) d \tau \\
= & \lim _{N^{\prime} \rightarrow \infty} \sum_{n^{\prime}=-1}^{N^{\prime}} \sum_{\alpha^{\prime}=-1}^{+1} \lim _{T_{1} \rightarrow \infty} \frac{1}{T_{1}} \int_{T_{0}}^{T_{0}+T_{1}} e^{\tau L} S_{1}^{0}\left(e^{-\alpha^{\prime} i \tau \sqrt{2 n^{\prime}+3}} P_{\alpha^{\prime}}^{\left(n^{\prime}\right)} \partial_{x} \overrightarrow{\mathcal{U}}\right) d \tau \\
= & \lim _{N^{\prime} \rightarrow \infty} \sum_{n^{\prime}=-1}^{N^{\prime}} \sum_{\alpha^{\prime}=-1}^{+1} \lim _{N \rightarrow \infty} \sum_{n=-1}^{N} \sum_{\alpha=-1}^{+1} \\
& \lim _{T_{1} \rightarrow \infty} \frac{1}{T_{1}} \int_{T_{0}}^{T_{0}+T_{1}} e^{i \tau\left(\alpha \sqrt{2 n+3}-\alpha^{\prime} \sqrt{2 n^{\prime}+3}\right)} d \tau P_{\alpha}^{(n)} S_{1}^{0} P_{\alpha^{\prime}}^{\left(n^{\prime}\right)} \partial_{x} \overrightarrow{\mathcal{U}} \\
= & P_{0} S_{1}^{0}\left(P_{0} \partial_{x} \overrightarrow{\mathcal{U}}\right)+\sum_{n=-1}^{+\infty} P_{1}^{(n)} S_{1}^{0}\left(P_{1}^{(n)} \partial_{x} \overrightarrow{\mathcal{U}}\right)+\sum_{n=-1}^{+\infty} P_{-1}^{(n)} S_{1}^{0}\left(P_{-1}^{(n)} \partial_{x} \overrightarrow{\mathcal{U}}\right) .
\end{aligned}
$$

The computation can be continued a little further:

$$
\begin{aligned}
P_{ \pm 1}^{(n)} S_{1}^{0} P_{ \pm 1}^{(n)} \partial_{x} \overrightarrow{\mathcal{U}} & =\left\langle\partial_{x} \overrightarrow{\mathcal{U}}, \vec{e}_{ \pm 1}^{n}\right\rangle\left\langle S_{1}^{0} \vec{e}_{ \pm 1}^{n}, \vec{e}_{ \pm 1}^{n}\right\rangle \vec{e}_{ \pm 1}^{n} \\
& =\left\langle S_{1}^{0} \vec{e}_{ \pm 1}^{n}, \vec{e}_{ \pm 1}^{n}\right\rangle P_{ \pm 1}^{(n)} \partial_{x} \overrightarrow{\mathcal{U}}
\end{aligned}
$$

and

$$
\begin{aligned}
\left\langle S_{1}^{0} \vec{e}_{ \pm 1}^{n}, \vec{e}_{ \pm 1}^{n}\right\rangle & =\frac{1}{2(2 n+3)} \int_{\mathbb{R}}\left(\begin{array}{c} 
\pm i \sqrt{n+2} \phi_{n+2} \\
\pm i \sqrt{n+1} \phi_{n} \\
0
\end{array}\right) \cdot\left(\begin{array}{c}
\mp i \sqrt{n+2} \phi_{n+2} \\
\pm i \sqrt{n+1} \phi_{n} \\
*
\end{array}\right) d y \\
& =\frac{1}{2(2 n+3)}\left((n+2)\left\langle\phi_{n+2}, \phi_{n+2}\right\rangle-(n+1)\left\langle\phi_{n}, \phi_{n}\right\rangle\right) \\
& =\frac{1}{2(2 n+3)}
\end{aligned}
$$


So

$$
\begin{aligned}
& \lim _{T_{1} \rightarrow \infty} \frac{1}{T_{1}} \int_{T_{0}}^{T_{0}+T_{1}} e^{\tau L} S_{1}^{0}\left(e^{-\tau L} \partial_{x} \overrightarrow{\mathcal{U}}\right) d \tau \\
= & P_{0} S_{1}^{0}\left(P_{0} \partial_{x} \overrightarrow{\mathcal{U}}\right)+\sum_{n=-1}^{+\infty} \frac{1}{2(2 n+3)}\left(P_{1}^{(n)}+P_{-1}^{(n)}\right) \partial_{x} \overrightarrow{\mathcal{U}} .
\end{aligned}
$$

Finally,

$$
\begin{aligned}
\lim _{T_{1} \rightarrow \infty} & \frac{1}{T_{1}} \int_{T_{0}}^{T_{0}+T_{1}} e^{\tau L} S_{2}\left(e^{-\tau L} \overrightarrow{\mathcal{U}}(t)\right) \partial_{y}\left(e^{-\tau L} \overrightarrow{\mathcal{U}}(t)\right) d \tau \\
= & \lim _{N \rightarrow \infty} \sum_{n^{\prime}, n^{\prime \prime}=-1}^{N} \sum_{\alpha^{\prime}, \alpha^{\prime \prime}=-1}^{+1} \lim _{T_{1} \rightarrow \infty} \frac{1}{T_{1}} \int_{T_{0}}^{T_{0}+T_{1}} e^{-i \tau\left(\alpha^{\prime} \sqrt{2 n^{\prime}+3}+\alpha^{\prime \prime} \sqrt{2 n^{\prime \prime}+3}\right)} \\
= & \left.\lim _{N \rightarrow \infty} \sum_{n^{\prime}, n^{\prime \prime}=-1} \sum_{\alpha^{\prime}, \alpha^{\prime \prime}=-1}^{N} \lim _{N^{\prime} \rightarrow \infty} \sum_{n=-1}^{N^{\prime}} \sum_{\alpha=-1}^{+1}{ }_{\alpha^{\prime}}^{+1} \overrightarrow{\mathcal{U}}(t)\right) \partial_{y}\left(P_{\alpha^{\prime \prime}}^{\left(n^{\prime \prime}\right)} \overrightarrow{\mathcal{U}}(t)\right) d \tau \\
& \lim _{T_{1} \rightarrow \infty} \frac{1}{T_{1}} \int_{T_{0}}^{T_{0}+T_{1}} e^{i \tau\left(\alpha \sqrt{2 n+3}-\alpha^{\prime} \sqrt{2 n^{\prime}+3}-\alpha^{\prime \prime} \sqrt{2 n^{\prime \prime}+3}\right)} d \tau \\
& P_{\alpha}^{(n)}\left(S_{2}\left(P_{\alpha^{\prime}}^{\left(n^{\prime}\right)} \overrightarrow{\mathcal{U}}(t)\right) \partial_{y}\left(P_{\alpha^{\prime \prime}}^{\left(n^{\prime \prime}\right)} \overrightarrow{\mathcal{U}}(t)\right)\right),
\end{aligned}
$$

after applying Lemma 3.3 several times. The factor $\alpha \sqrt{2 n+3}-\alpha^{\prime} \sqrt{2 n^{\prime}+3}-$ $\alpha^{\prime \prime} \sqrt{2 n^{\prime \prime}+3}$ in the exponential vanishes only in the following cases:

$$
\begin{gathered}
\alpha=\alpha^{\prime}=\alpha^{\prime \prime}=0 \\
\alpha=0, \quad \alpha^{\prime}=-\alpha^{\prime \prime} \neq 0, \quad n^{\prime}=n^{\prime \prime} \\
\alpha^{\prime}=0, \quad \alpha=\alpha^{\prime \prime} \neq 0, \quad n=n^{\prime \prime} \\
\alpha^{\prime \prime}=0, \quad \alpha=\alpha^{\prime} \neq 0, \quad n=n^{\prime} .
\end{gathered}
$$

The terms corresponding to (3.39) and (3.40), however, don't contribute anything to the limit. Because the third component of $P_{0}^{(m)}$ applied to something is zero, for all $m$, those corresponding to (3.39) are all identically zero:

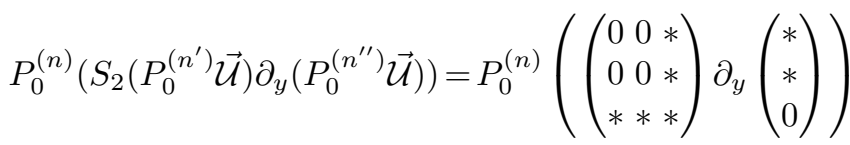

$$
\begin{aligned}
& =P_{0}^{(n)}\left(\begin{array}{l}
0 \\
0 \\
*
\end{array}\right)=0 .
\end{aligned}
$$

On the other hand, those corresponding to (3.40) cancel out by pairs, because

$$
\begin{aligned}
P_{0}^{(n)}( & \left.S_{2}\left(P_{ \pm 1}^{\left(n^{\prime}\right)} \overrightarrow{\mathcal{U}}\right) \partial_{y}\left(P_{\mp 1}^{\left(n^{\prime \prime}\right)} \overrightarrow{\mathcal{U}}\right)\right)=\left\langle\overrightarrow{\mathcal{U}}, \vec{e}_{1}^{n^{\prime}}\right\rangle\left\langle\overrightarrow{\mathcal{U}}, \vec{e}_{-1}^{n^{\prime}}\right\rangle P_{0}^{(n)}\left(S_{2}\left(\vec{e}_{ \pm 1}^{n^{\prime}}\right) \partial_{y} \vec{e}_{\mp 1}^{n^{\prime}}\right) \\
= & \frac{ \pm i}{8 \sqrt{2 n+3}}\left\langle\overrightarrow{\mathcal{U}}, \vec{e}_{1}^{n^{\prime}}\right\rangle\left\langle\mathcal{\mathcal { U }}, \vec{e}_{-1}^{n^{\prime}}\right\rangle \\
& P_{0}^{(n)}\left(\begin{array}{c}
-4 \sqrt{n+2} \phi_{n+1} \phi_{n+2}^{\prime}+\left(\sqrt{n+2} \phi_{n+2}-\sqrt{n+1} \phi_{n}\right) \phi_{n+1}^{\prime} \\
4 \sqrt{n+1} \phi_{n+1} \phi_{n}^{\prime}+\left(\sqrt{n+2} \phi_{n+2}-\sqrt{n+1} \phi_{n}\right) \phi_{n+1}^{\prime} \\
*
\end{array}\right) .
\end{aligned}
$$


Hence,

$$
\begin{aligned}
& \lim _{T_{1} \rightarrow \infty} \frac{1}{T_{1}} \int_{T_{0}}^{T_{0}+T_{1}} e^{\tau L} S_{2}\left(e^{-\tau L} \overrightarrow{\mathcal{U}}\right) \partial_{y}\left(e^{-\tau L} \overrightarrow{\mathcal{U}}\right) d \tau \\
&=\sum_{n=-1}^{+\infty} P_{1}^{(n)}\left(S_{2}\left(P_{0} \overrightarrow{\mathcal{U}}\right) \partial_{y}\left(P_{1}^{(n)} \overrightarrow{\mathcal{U}}\right)+S_{2}\left(P_{1}^{(n)} \overrightarrow{\mathcal{U}}\right) \partial_{y}\left(P_{0} \overrightarrow{\mathcal{U}}\right)\right) \\
& \quad+\sum_{n=-1}^{+\infty} P_{-1}^{(n)}\left(S_{2}\left(P_{0} \overrightarrow{\mathcal{U}}\right) \partial_{y}\left(P_{-1}^{(n)} \overrightarrow{\mathcal{U}}\right)+S_{2}\left(P_{-1}^{(n)} \overrightarrow{\mathcal{U}}\right) \partial_{y}\left(P_{0} \overrightarrow{\mathcal{U}}\right)\right) \\
&=\sum_{n=-1}^{+\infty}\left(\left\langle S_{2}\left(P_{0} \overrightarrow{\mathcal{U}}\right) \partial_{y} \vec{e}_{1}^{n}+S_{2}\left(\vec{e}_{1}^{n}\right) \partial_{y} P_{0} \overrightarrow{\mathcal{U}}, \vec{e}_{1}^{n}\right\rangle P_{1}^{(n)} \overrightarrow{\mathcal{U}}\right. \\
&+\left\langle S_{2}\left(P_{0} \overrightarrow{\mathcal{U}}\right) \partial_{y} \vec{e}_{-1}^{n}+S_{2}\left(\vec{e}_{-1}^{n}\right) \partial_{y} P_{0} \overrightarrow{\mathcal{U}}, \vec{e}_{-1}^{n}\right\rangle P_{-1}^{(n)} \overrightarrow{\mathcal{U}}
\end{aligned}
$$

\section{Concluding discussion}

The theorems and proofs presented above pave the way for a rigorous PDE analysis of both the equatorial shallow water equations and the equatorial Boussinesq equations in other suitable physically relevant singular limit regimes [18, 20, 2]. These results will be reported elsewhere by the authors in the near future. The extension of the present results to include the active nonlinear effects of moisture [7] requires new a priori estimates beyond those developed in [7] and the present paper.

As the limit system (LW) is linear, its solutions are global in time and so it is expected that, given any time $T>0$, the solutions of $\left(\mathrm{SW}_{\epsilon}\right)$ exist on $[0, T]$ for all $\epsilon$ smaller than some $\epsilon_{0}=\epsilon_{0}(T)$. When the initial data are well prepared, a reasoning by Iftimie [9] (see also [4]), solving this problem for the quasigeostrophic limit, can be adapted; it yields $1 / \epsilon_{0} \sim \exp (\exp C T)$ assuming that the oscillating parts of the data are of order $\epsilon$. When the initial data are not well prepared, the method of Schochet [25, Theorem 2.3] still allows us to prove that the times of existence of (SW $\epsilon$ ) tend to infinity, but then we have no estimate on $\epsilon_{0}$. These results will be included in another paper.

Finally, it is an interesting challenge to see whether there is a direct proof of convergence in the weak topology through only the basic $L^{2}$ energy estimate alone. Masmoudi and Lions [14, 22] (see also [13]) have obtained such results for the compressible to incompressible fluid limit with fixed viscosity under suitable hypotheses. However, in the physical problems of tropical meteorology and oceanography, the dissipative effects are (undifferentiated) Rayleigh damping and friction $[16,7]$ so completely different ideas are needed; on the other hand, as discussed here, the formal limit equation is linear so this might help such a strategy in the weak topology.

Acknowledgements. Alexandre Dutrifoy thanks the Département des Relations Internationales de l'Université Libre de Bruxelles for funding his stay at the Courant Institute. Andrew Majda thanks the National Science Foundation and Office of Naval Research for generous support of this research.

\section{REFERENCES}

[1] J. A. Biello and A. J. Majda, The effect of meridional and vertical shear on the interaction of equatorial baroclinic and barotropic Rossby waves, Stud. Appl. Math., 112(4), 341-390, 2004. 
[2] J. A. Biello and A. J. Majda, A new multiscale model for the Madden-Julian oscillation, J. Atmospheric Sci., 62(6), 1694-1721, 2005.

[3] A. J. Bourgeois and J. T. Beale, Validity of the quasigeostrophic model for large-scale flow in the atmosphere and ocean, SIAM J. Math. Anal., 25(4), 1023-1068, 1994.

[4] J.-Y. Chemin, À propos d'un problème de pénalisation de type antisymétrique, J. Math. Pures Appl., 76(9), 739-755, 1997.

[5] P. Embid and A. Majda, Averaging over fast gravity waves for geophysical flows with arbitrary potential vorticity, Comm. Partial Differential Equations, 21(3-4), 619-658, 1996.

[6] P. F. Embid and A. J. Majda, Low Froude number limiting dynamics for stably stratified flow with small or finite Rossby numbers, Geophys. Astrophys. Fluid Dynam., 87(1-2), 1-50, 1998.

[7] D. M. W. Frierson, A. J. Majda and O. M. Pauluis, Large scale dynamics of precipitation fronts in the tropical atmosphere: a novel relaxation limit, Comm. Math. Sci., 2(4), 591-626, 2004.

[8] A. E. Gill, Atmosphere-ocean Dynamics, Academic Press, New York, 1982.

[9] D. Iftimie, Approximation of the quasigeostrophic system with the primitive systems, Asymptot. Anal., 21(2), 89-97, 1999.

[10] J. L. Joly, G. Métivier and J. Rauch, Coherent and focusing multidimensional nonlinear geometric optics, Ann. Sci. École Norm. Sup. 4, 1(28), 51-113, 1995.

[11] S. Klainerman and A. Majda, Singular limits of quasilinear hyperbolic systems with large parameters and the incompressible limit of compressible fluids, Comm. Pure Appl. Math., 34(4), 481-524, 1981.

[12] S. Klainerman and A. Majda, Compressible and incompressible fluids, Comm. Pure Appl. Math., 35(5), 629-651, 1982.

[13] P.-L. Lions and N. Masmoudi, Incompressible limit for a viscous compressible fluid, J. Math. Pures Appl., 77(6), 585-627, 1998.

[14] P.-L. Lions and N. Masmoudi, Une approche locale de la limite incompressible, C. R. Acad. Sci. Paris Sér. I Math., 329(5), 387-392, 1999

[15] A. Majda, Compressible Fluid Flow and Systems of Conservation Laws in Several Space Variables, Applied Mathematical Sciences, Springer-Verlag, New York, 53, 1984.

[16] A. Majda, Introduction to PDEs and Waves for the Atmosphere and Ocean, Courant Lecture Notes in Mathematics, AMS/CIMS, New York, 9, 2003.

[17] A. Majda and P. Embid, Averaging over fast gravity waves for geophysical flow with unbalanced initial data, Theoret. Comput. Fluid Dynam., 11, 155-169, 1998.

[18] A. Majda and R. Klein, Systematic multiscale models for the tropics, J. Atmospheric Sci., 60, 393-408, 2003.

[19] A. J. Majda and J. A. Biello, The nonlinear interaction of barotropic and equatorial baroclinic Rossby waves, J. Atmospheric Sci., 60(15), 1809-1821, 2003.

[20] A. J. Majda and J. A. Biello, A multiscale model for tropical intraseasonal oscillations, Proc. Natl. Acad. Sci. USA, 101(14), 4736-4741, (electronic), 2004.

[21] A. J. Majda, R. R. Rosales, E. G. Tabak and C. V. Turner, Interaction of large-scale equatorial waves and dispersion of Kelvin waves through topographic resonances, J. Atmospheric Sci., 56(24), 4118-4133, 1999.

[22] N. Masmoudi, Incompressible, inviscid limit of the compressible Navier-Stokes system, Ann. Inst. H. Poincaré Anal. Non Linéaire, 18(2), 199-224, 2001.

[23] J. Pedlosky, Geophysical Fluid Dynamics, Springer, New York, 1979.

[24] S. G. Philander, El Niño, La Niña, and The Southern Oscillation, Academic Press, San Diego, 1990.

[25] S. Schochet, Fast singular limits of hyperbolic PDEs, J. Differential Equations, 114(2), 476-512, 1994.

[26] S. Schochet, Resonant nonlinear geometric optics for weak solutions of conservation laws, J. Differential Equations, 113(2), 473-504, 1994.

[27] T. Sideris, The lifespan of smooth solutions to the three-dimensional compressible Euler equations and the incompressible limit, Indiana Univ. Math. J., 40(2), 535-550, 1991.

[28] R. K. Smith, editor, The physics and parametrization of moist atmospheric convection, NATO Advanced Study Institute Series C. Mathematical and Physical Sciences, Kluwer, Norwell, Mass., 505, 1997. 